\title{
Potential Solar Sail Degradation Effects on Trajectory and Attitude Control
}

\author{
Bernd Dachwald* and Wolfgang Seboldt ${ }^{\dagger}$ \\ DLR, German Aerospace Center, 51170 Cologne, Germany \\ Malcolm Macdonald ${ }^{\ddagger}$ \\ University of Glasgow, G12 8QQ Glasgow, Scotland \\ Giovanni Mengali ${ }^{\S}$ and Alessandro A. Quarta \\ University of Pisa, 56122 Pisa, Italy \\ Colin R. Mc Innes ${ }^{\|}$ \\ University of Strathclyde, G1 1XJ Glasgow, Scotland \\ Leonel Rios-Reyes** and Daniel J. Scheeres ${ }^{\dagger \dagger}$ \\ University of Michigan, Ann Arbor, MI 48105, USA \\ Bong Wie \\ Arizona State University, Tempe, AZ 85287, USA \\ Marianne Görlich and Franz Lura \\ DLR, German Aerospace Center, 12489 Berlin, Germany \\ Benjamin Diedrich \\ NOAA, Silver Spring, ML 20910, USA \\ Volodymyr Baturkin \\ National Technical University of Ukraine, 03056 Kiev, Ukraine \\ Victoria L. Coverstone \\ University of Illinois at Urbana-Champaign, Urbana, IL 61801, USA \\ Manfred Leipold \\ Kayser-Threde GmbH, 81379 Munich, Germany \\ Gregory P. Garbe \\ NASA Marshal Space Flight Center, Huntsville, AL 85812, USA
}

\footnotetext{
*Scientist, Institute of Space Simulation, bernd.dachwald@dlr.de, Member AIAA.

†Senior Scientist, Institute of Space Simulation, wolfgang.seboldt@dlr.de.

$\ddagger$ Research Assistant, Dept. of Aerospace Engineering, m.macdonald@aero.gla.ac.uk.

$\S$ Associate Professor, Dept. of Aerospace Engineering, g.mengali@ing.unipi.it.

『Research Assistant, Dept. of Aerospace Engineering, a.quarta@ing.unipi.it.

\| Professor, Dept. of Mechanical Engineering, colin.mcinnes@strath.ac.uk, Member AIAA.

**Graduate Student, Dept. of Aerospace Engineering, leonelr@umich.edu, Student Member AIAA.

$\dagger^{\dagger}$ Associate Professor, Dept. of Aerospace Engineering, scheeres@umich.edu, Associate Fellow AIAA.

¥¥Professor, Dept. of Mechanical \& Aerospace Engineering, bong.wie@asu.edu, Associate Fellow AIAA.
} 
The optical properties of the thin metalized polymer films that are projected for solar sails are assumed to be affected by the erosive effects of the space environment. Their degradation behavior in the real space environment, however, is to a considerable degree indefinite, because initial ground test results are controversial and relevant in-space tests have not been made so far. The standard optical solar sail models that are currently used for trajectory and attitude control design do not take optical degradation into account, hence its potential effects on trajectory and attitude control have not been investigated so far. Nevertheless, optical degradation is important for high-fidelity solar sail mission analysis, because it decreases both the magnitude of the solar radiation pressure force acting on the sail and also the sail control authority. Therefore, we propose a simple parametric optical solar sail degradation model that describes the variation of the sail film's optical coefficients with time, depending on the sail film's environmental history, i.e., the radiation dose. The primary intention of our model is not to describe the exact behavior of specific film-coating combinations in the real space environment, but to provide a more general parametric framework for describing the general optical degradation behavior of solar sails. Using our model, the effects of different optical degradation behaviors on trajectory and attitude control are investigated for various exemplary missions.

\section{Introduction}

The objective of this paper is to establish a parametric model for optical solar sail degradation and to describe its effects on trajectory and attitude control. Solar sails require large highly reflective ultralightweight surfaces. The optical and mechanical properties of the thin metalized polymer films that are projected for solar sails are assumed to be affected by the erosive effects of the space environment. Optical solar sail degradation (OSSD) should be considered for a thorough solar sail mission analysis because it decreases not only the magnitude of the solar radiation pressure (SRP) force acting on the sail but also the sail control authority, because it reduces the magnitude of the SRP force component perpendicular to the sun-sail direction. Mechanical solar sail degradation (MSSD) should be considered in the solar sail design process, because it decreases the tensile strength of the sail film and is likely to affect the sail performance. MSSD, however, is not considered within this paper because its consequences depend on the actual mechanical strain in the sail film and, therefore, on the actual solar sail design.

Although much ground and space testing has been done to measure the optical degradation of metalized polymer films as second surface mirrors (metalized on the back side), to our knowledge, no systematic testing to measure the optical degradation of candidate solar sail films (metalized on the front side) on ground or in space has been reported so far, so that the optical degradation behavior (and therefore the degradation behavior of the propulsive capability) of solar sails in the real space environment is to a considerable degree indefinite. Nevertheless, solar sail mission designers necessitate an OSSD model to estimate the potential effects of OSSD on their missions.

The standard optical solar sail model is the non-perfectly reflecting sail model ${ }^{\mathrm{a}}$, as it is described, e.g., in Refs. 1, 2, or 3. Based on this model, Rios-Reyes and Scheeres developed a generalized sail model for non-flat solar sails, which allows one to model also local variations of the optical sail properties. ${ }^{4}$ Both models do not take OSSD into account. Therefore, the authors of this paper established in November 2004 a "Solar Sail Degradation Model Working Group" (SSDMWG) with the aim to make the next step towards a realistic high-fidelity optical solar sail model by elaborating a parametric model for OSSD. Our OSSD model describes the variation of the sail film's optical coefficients with time, depending on the sail film's environmental history, i.e., the radiation dose. The primary intention of this model is not to describe the exact behavior of specific film-coating combinations in the real space environment, but to provide a more general parametric framework for the general optical degradation behavior of solar sails. It should later be refined, when results from ground and in-space tests have become available. Until then, our model can be used to investigate the effects of different potential degradation behaviors on various mission aspects, e.g. trajectory optimization and attitude control, by varying the OSSD model parameters.

The paper is organized as follows. Section II reviews the SRP force models for non-perfectly reflecting solar sails. In section III, a parametric OSSD model is proposed that describes the variation of the sail film's optical coefficients with time, depending on the sail film's environmental history, i.e., the radiation dose. This model is then used in section IV to estimate the effects of OSSD on trajectory optimization and

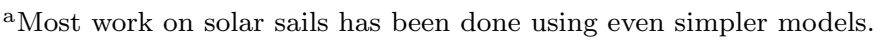


attitude control for five exemplary missions: a Mars rendezvous, a Mercury rendezvous, a fast Neptune flyby, a fast transfer to the heliopause (200 AU), and, finally, for artificial Lagrange-point missions.

\section{The Non-Perfectly Reflecting Solar Sail}

For the description of the solar radiation pressure (SRP) force exerted on a solar sail, it is convenient to introduce two unit vectors. The first one is the sail normal vector $\boldsymbol{n}$, which is perpendicular to the sail surface and always directed away from the sun. In the orbit frame ${ }^{\mathrm{b}} \mathcal{O}^{3}$, its direction, which describes the sail attitude, is expressed by the pitch angle $\alpha$ and the clock angle $\delta$ (Fig. 1). The second unit vector is the thrust unit vector $\boldsymbol{m}$, which points along the direction of the SRP force. Its direction is described likewise by the cone angle $\theta$ and the clock angle $\delta$.

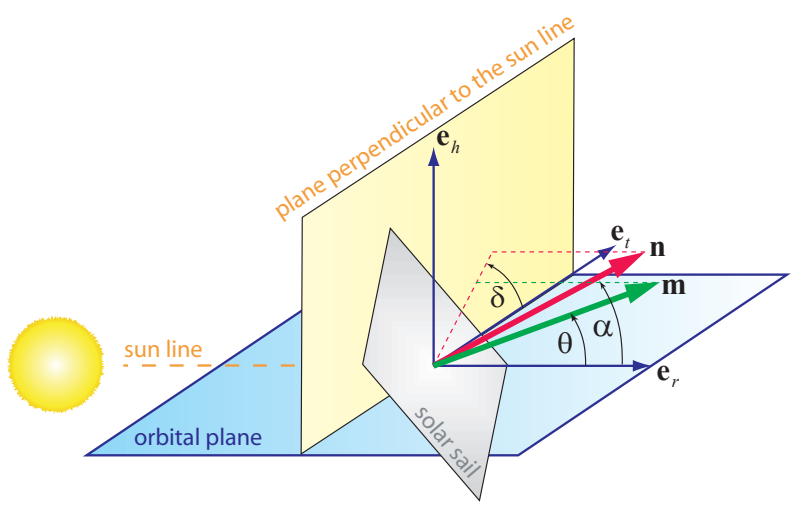

Figure 1. Definition of the sail normal vector and the thrust normal vector

At a distance $r$ from the sun, the SRP is

$$
P=\frac{S_{0}}{c}\left(\frac{r_{0}}{r}\right)^{2}=P_{0} \cdot\left(\frac{r_{0}}{r}\right)^{2}=4.563 \frac{\mu \mathrm{N}}{\mathrm{m}^{2}} \cdot\left(\frac{r_{0}}{r}\right)^{2}
$$

where $S_{0}=1368 \mathrm{~W} / \mathrm{m}^{2}$ is the solar constant, $c$ is the speed of light in vacuum, and $r_{0}$ is 1 astronomical unit (1 AU).

Different levels of simplification for the optical characteristics of a solar sail result in different models for the magnitude and direction of the SRP force acting on the sail. The most simple model assumes an ideally reflecting sail surface. It will here be denoted as model IR (ideal reflection). It can be easily derived ${ }^{\mathrm{c}}$ (see also Fig. 2(a)) that the SRP force exerted on an ideal sail is ${ }^{\mathrm{d}}$

$$
\boldsymbol{F}_{\mathrm{SRP}}=2 P A \cos \alpha \cos \alpha \boldsymbol{n}
$$

Thus, for model IR, the SRP force is always along the direction of the sail normal vector, $\boldsymbol{m} \equiv \boldsymbol{n}$.

\section{A. The Non-Perfectly Reflecting Solar Sail Model}

For a thorough attitude control and trajectory simulation, a more sophisticated SRP force model than IR must be employed, which takes into account the optical coefficients of the real sail film. This model will here be denoted as model NPR (non-perfect reflection). It was proposed in the 1970s for solar sail trajectory optimization by Sauer ${ }^{5}$ and further studied by Forward, ${ }^{1}$ but found little application until recently. ${ }^{6-10}$

\footnotetext{
${ }^{\mathrm{b}} \mathcal{O}^{3}=\left\{\boldsymbol{e}_{r}, \boldsymbol{e}_{t}, \boldsymbol{e}_{h}\right\}$ is an orthogonal right-handed polar coordinate frame. $\boldsymbol{e}_{r}$ points always along the sun-spacecraft line, $\boldsymbol{e}_{h}$ is the orbit plane normal (pointing along the spacecraft's orbital angular momentum vector), and $\boldsymbol{e}_{t}$ completes the right-handed coordinate system $\left(\boldsymbol{e}_{r} \times \boldsymbol{e}_{t}=\boldsymbol{e}_{h}\right)$.

${ }^{\mathrm{c}}$ See e.g. Ref. 3 pp. 38-39.

d Note that one $\cos \alpha$ results from the projection of the sail area onto $\boldsymbol{e}_{r}$, whereas the other $\cos \alpha$ results from the projection of the two SRP force components onto $\boldsymbol{n}$.
} 


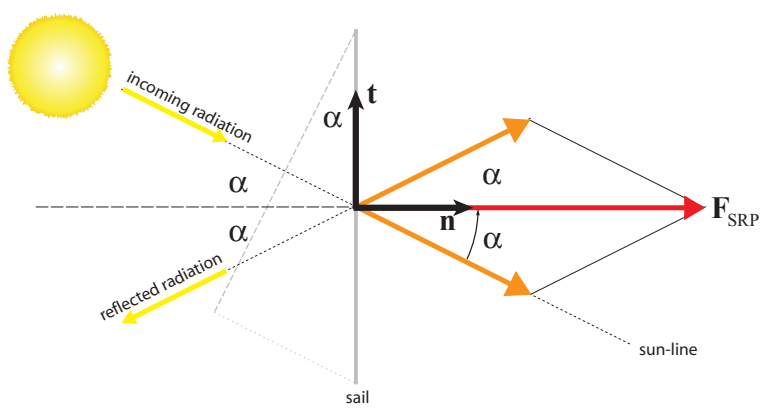

(a) on an ideal sail

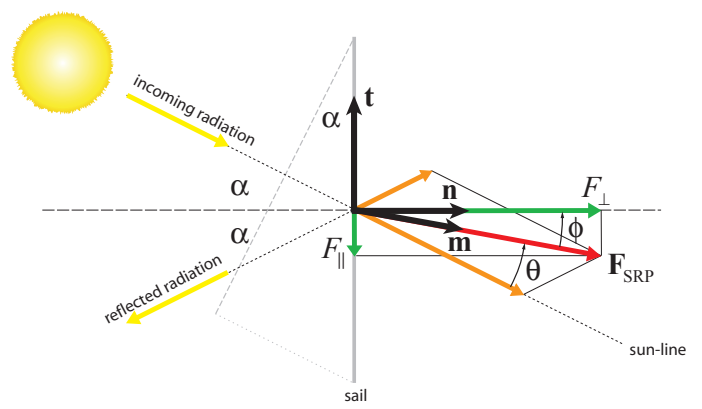

(b) on a non-perfectly reflecting sail

Figure 2. SRP force

\section{Optical Solar Sail Coefficients}

In model NPR, the optical characteristics of the sail film are parameterized by the absorption coefficient $a$, the reflection coefficient $\rho$, the transmission coefficient $\tau$, and the emission coefficient $\varepsilon$, with the constraint $a+\rho+\tau=1$. Assuming $\tau=0$ for the reflecting side of the solar sail, the absorption coefficient is $a=1-\rho$. Because not all photons are reflected specularly, the reflection coefficient can be further divided into a specular reflection coefficient $\rho_{s}$, a diffuse reflection coefficient $\rho_{d}$, and a back reflection coefficient $\rho_{b}$, with the constraint $\rho_{s}+\rho_{d}+\rho_{b}=\rho$. Assuming $\rho_{b}=0$, this can also be expressed by introducing a specular reflection factor $s$, so that $s=\rho_{s} / \rho \Leftrightarrow \rho_{s}=s \rho \Rightarrow \rho_{d}=(1-s) \rho$. The emission coefficient $\varepsilon$ describes the power $W$ that is emitted from a surface of area $A$ at absolute temperature $T, W=A \varepsilon \sigma T^{4}$, where $\sigma=5.67 \cdot 10^{-8} \mathrm{Wm}^{-2} \mathrm{~K}^{-4}$ is the Stefan-Boltzmann constant. The emission coefficients of the sail's front and back side are $\varepsilon_{\mathrm{f}}$ and $\varepsilon_{\mathrm{b}}$, respectively. The angular distribution of the emitted and the diffusely reflected photons is described by the non-Lambertian coefficients of the sail's front and back side, $B_{\mathrm{f}}$ and $B_{\mathrm{b}}$, respectively. Thus, model NPR parameterizes the optical characteristics of the sail film by the following set of optical coefficients: $\mathcal{P}=\left\{\rho, s, \varepsilon_{\mathrm{f}}, \varepsilon_{\mathrm{b}}, B_{\mathrm{f}}, B_{\mathrm{b}}\right\}$. According to Wright, the optical coefficients for a sail with a highly reflective aluminum-coated front side and a highly emissive chromium-coated back side ${ }^{\mathrm{e}}$ are $\mathcal{P}_{\mathrm{Al} \mid \mathrm{Cr}}=\left\{\rho=0.88, s=0.94, \varepsilon_{\mathrm{f}}=0.05, \varepsilon_{\mathrm{b}}=0.55, B_{\mathrm{f}}=0.79, B_{\mathrm{b}}=0.55\right\} .^{2}$

\section{Solar Radiation Pressure Force}

Using the optical sail coefficients defined above, it can be shown ${ }^{\mathrm{f}}$ that in a sail-fixed $2 \mathrm{D}^{\mathrm{g}}$ coordinate frame $\mathcal{S}=\{\boldsymbol{n}, \boldsymbol{t}\}$, the SRP force exerted on the solar sail has a normal component $F_{\perp}$ (along $\boldsymbol{n}$ ) and a transverse component $F_{\|}$(along $\boldsymbol{t}$, see Fig. 2(b)) with

$$
\begin{aligned}
F_{\perp} & =\boldsymbol{F}_{\mathrm{SRP}} \cdot \boldsymbol{n}=2 P A \cos \alpha \psi_{\perp} \\
F_{\|} & =\boldsymbol{F}_{\mathrm{SRP}} \cdot \boldsymbol{t}=-2 P A \cos \alpha \psi_{\|}
\end{aligned}
$$

where

$$
\psi_{\perp} \triangleq \frac{1}{2}(1+s \rho) \cos \alpha+\frac{1}{2}\left[B_{\mathrm{f}}(1-s) \rho+(1-\rho) \frac{\varepsilon_{\mathrm{f}} B_{\mathrm{f}}-\varepsilon_{\mathrm{b}} B_{\mathrm{b}}}{\varepsilon_{\mathrm{f}}+\varepsilon_{\mathrm{b}}}\right] \quad \psi_{\|} \triangleq \frac{1}{2}(1-s \rho) \sin \alpha
$$

Three characteristic optical sail coefficients may be defined to simplify Eq. (4):

$$
a_{1} \triangleq \frac{1}{2}(1+s \rho) \quad a_{2} \triangleq \frac{1}{2}\left[B_{\mathrm{f}}(1-s) \rho+(1-\rho) \frac{\varepsilon_{\mathrm{f}} B_{\mathrm{f}}-\varepsilon_{\mathrm{b}} B_{\mathrm{b}}}{\varepsilon_{\mathrm{f}}+\varepsilon_{\mathrm{b}}}\right] \quad a_{3} \triangleq \frac{1}{2}(1-s \rho)
$$

so that

$$
\psi_{\perp}=a_{1} \cos \alpha+a_{2} \quad \psi_{\|}=a_{3} \sin \alpha
$$

${ }^{\mathrm{e}}$ This is to keep the sail temperature at a moderate limit, as we will see later from Eq. (15).

${ }^{\text {f}}$ See e.g. Ref. 3 pp. 47-49 for derivation.

gBecause of symmetry, the third dimension is not relevant here. 
The total SRP force vector may then be written as

$$
\boldsymbol{F}_{\mathrm{SRP}}=\left(F_{\perp}^{2}+F_{\|}^{2}\right)^{1 / 2} \boldsymbol{m}=2 P A \cos \alpha\left(\psi_{\perp}^{2}+\psi_{\|}^{2}\right)^{1 / 2} \boldsymbol{m}
$$

or

$$
\boldsymbol{F}_{\mathrm{SRP}}=2 P A \cos \alpha \Psi \boldsymbol{m}
$$

where $\Psi \triangleq\left(\psi_{\perp}^{2}+\psi_{\|}^{2}\right)^{1 / 2}$ depends only on the pitch angle $\alpha$ and the optical coefficients $\mathcal{P}$ of the sail film. Note the symmetry between Eqs. (2) and (8). The angle between $\boldsymbol{m}$ and $\boldsymbol{e}_{r}$ is the cone angle $\theta$ and the angle between $\boldsymbol{m}$ and $\boldsymbol{n}$ is called centerline angle $\phi$. It may be calculated via

$$
\phi=\arctan \left(\frac{\psi_{\|}}{\psi_{\perp}}\right)
$$

From Eq. (9), the cone angle is then obtained as

$$
\theta=\alpha-\phi=\alpha-\arctan \left(\frac{\psi_{\|}}{\psi_{\perp}}\right)
$$

The SRP force can also be written in components along the unit vectors of a $2 \mathrm{D}$ orbit frame $\mathcal{O}^{2}=\left\{\boldsymbol{e}_{r}, \boldsymbol{e}_{t}\right\}$, a decomposition that will be used in the next section to determine which optical coefficients $p \in \mathcal{P}$ are the most important ones for OSSD modeling. The $\mathcal{O}^{2}$-frame components of $\boldsymbol{F}_{\mathrm{SRP}}$ can be obtained simply from the $\mathcal{S}$-frame components via

$$
\left(\begin{array}{c}
F_{r} \\
F_{t}
\end{array}\right)=\left[\begin{array}{cc}
\cos \alpha & -\sin \alpha \\
\sin \alpha & \cos \alpha
\end{array}\right]\left(\begin{array}{l}
F_{\perp} \\
F_{||}
\end{array}\right)
$$

so that

$$
\begin{aligned}
& F_{r}=\boldsymbol{F}_{\mathrm{SRP}} \cdot \boldsymbol{e}_{r}=2 P A \cos \alpha\left(a_{1} \cos ^{2} \alpha+a_{2} \cos \alpha+a_{3} \sin ^{2} \alpha\right) \\
& F_{t}=\boldsymbol{F}_{\mathrm{SRP}} \cdot \boldsymbol{e}_{t}=2 P A \cos \alpha \sin \alpha\left(a_{1} \cos \alpha+a_{2}-a_{3} \cos \alpha\right)
\end{aligned}
$$

Finally, the SRP force can be written in components along $\boldsymbol{e}_{r}$ and $\boldsymbol{n},{ }^{10}$ a decomposition that will be used later for the derivation of the optimal control law (section IV.A). The result is:

$$
\boldsymbol{F}_{\mathrm{SRP}}=2 P A \cos \alpha\left[b_{1} \boldsymbol{e}_{r}+\left(b_{2} \cos \alpha+b_{3}\right) \boldsymbol{n}\right]
$$

where the coefficients $b_{1}, b_{2}$, and $b_{3}$ are defined as

$$
b_{1} \triangleq \frac{1}{2}(1-s \rho) \quad b_{2} \triangleq s \rho \quad b_{3} \triangleq \frac{1}{2}\left[B_{\mathrm{f}}(1-s) \rho+(1-\rho) \frac{\varepsilon_{\mathrm{f}} B_{\mathrm{f}}-\varepsilon_{\mathrm{b}} B_{\mathrm{b}}}{\varepsilon_{\mathrm{f}}+\varepsilon_{\mathrm{b}}}\right]
$$

\section{Solar Sail Temperature}

The equilibrium temperature of the solar sail is ${ }^{\mathrm{h}}$

$$
T=\left[\frac{S_{0}}{\sigma} \frac{a}{\varepsilon_{\mathrm{f}}+\varepsilon_{\mathrm{b}}}\left(\frac{r_{0}}{r}\right)^{2} \cos \alpha\right]^{1 / 4} \propto \frac{\cos ^{1 / 4} \alpha}{r^{1 / 2}} \Rightarrow r_{\min } \propto \frac{\cos ^{1 / 2} \alpha}{T_{\lim }^{2}}
$$

Thus, $T=T(r, \alpha, \mathcal{P})$. Eq. (15) shows that, for a given sail film-coating combination, there is a minimum sun-sail distance $r_{\min }$, which depends not only on the sail film temperature limit $T_{\text {lim }}$, but also on the pitch angle $\alpha$.

\footnotetext{
${ }^{\mathrm{h}}$ See e.g. Ref. 3 pp. 48-49 for derivation.
} 


\section{Optical SRP Force Sensitivity}

Using the SRP force on a black body with an area $A$ perpendicular to the incoming radiation, $P A$, one can normalize $F_{r}$ and $F_{t}, f_{r}=F_{r} / P A$ and $f_{t}=F_{t} / P A$. The sensitivity of $f_{r}$ and $f_{t}$ with respect to the different optical coefficients $p$ can then be described by

$$
\frac{\partial f_{r}}{\partial p}=\cos \alpha\left(P_{p 1} \cos \alpha+P_{p 2} \cos 2 \alpha\right) \quad \frac{\partial f_{t}}{\partial p}=\cos \alpha \sin \alpha\left(P_{p 1}+2 P_{p 2} \cos \alpha\right)
$$

For the optical parameters of an Al|Cr-coated sail, one gets $P_{\rho 1}=0.4857 \overline{3}, P_{\rho 2}=0.94, P_{s 1}=-0.6952$, $P_{s 2}=0.88, P_{\varepsilon_{\mathrm{f}} 1}=0.245 \overline{6}, P_{\varepsilon_{\mathrm{b}} 1}=-0.022 \overline{3}, P_{B_{\mathrm{f}} 1}=0.0628, P_{B_{\mathrm{b}} 1}=-0.11, P_{\varepsilon_{\mathrm{f}} 2}=P_{\varepsilon_{\mathrm{b}} 2}=P_{B_{\mathrm{f}} 2}=P_{B_{\mathrm{b}} 2}=0$. Fig. 3 shows how the SRP force components $f_{t}$ and $f_{r}$ vary with $\alpha$. Note that the sensitivity of $f_{t}$ and $f_{r}$ is largest with respect to $\rho, s$, and $\varepsilon_{\mathrm{f}}$. The sensitivity with respect to the other optical coefficients is only minor and could be neglected for a first OSSD analysis.

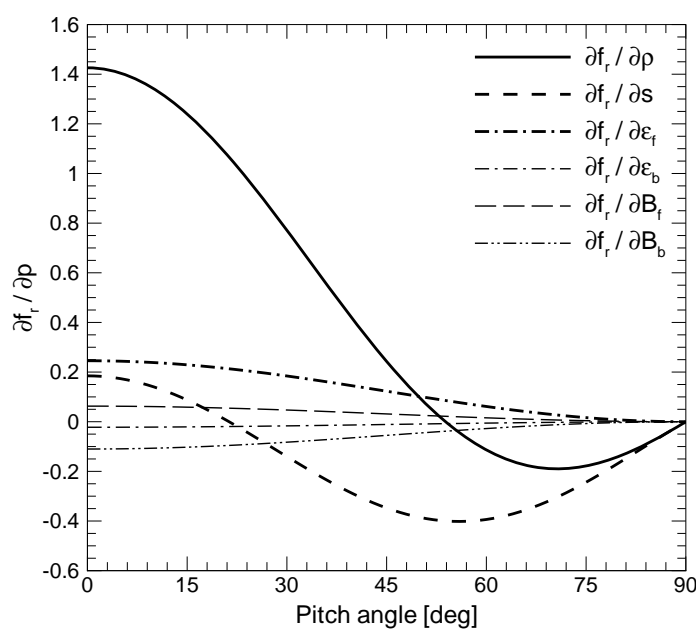

(a) $\partial f_{r} / \partial p$

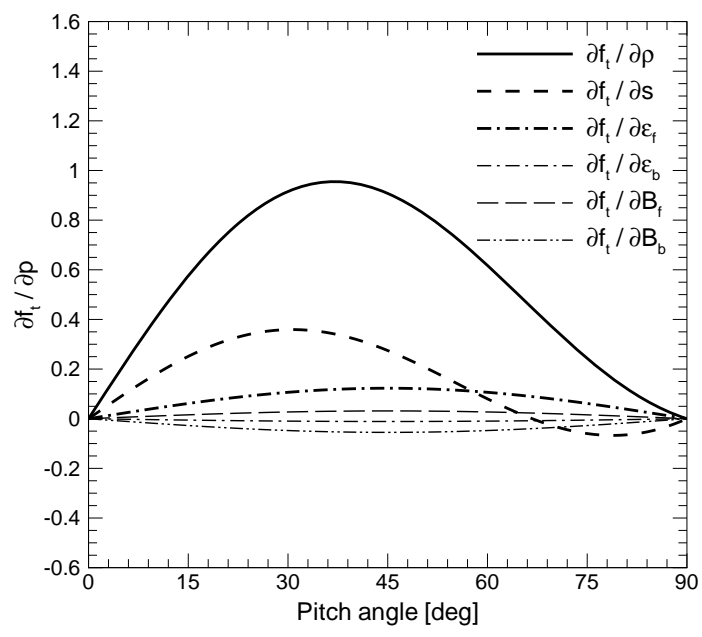

(b) $\partial f_{t} / \partial p$

Figure 3. Sensitivity of the SRP force components w.r.t. the optical coefficients

\section{B. The Simplified Non-Perfectly Reflecting Solar Sail Model}

To simplify model NPR, one may assume specular reflectivity of the sail, $s=1$, and equal products of the emission coefficient and the non-Lambertian coefficient for the front and back side, $\varepsilon_{\mathrm{f}} B_{\mathrm{f}}=\varepsilon_{\mathrm{b}} B_{\mathrm{b}}$. This model will here be denoted as model SNPR (simplified non-perfect reflection). Within model SNPR, the symbol $\eta$ denotes the reflection coefficient. The SRP force components in the $\mathcal{S}$-frame are in this case

$$
\begin{aligned}
F_{\perp} & =2 P A \cos \alpha \psi_{\perp} \\
F_{||} & =-2 P A \cos \alpha \psi_{\|}
\end{aligned}
$$

with

$$
\psi_{\perp} \triangleq \frac{1}{2}(1+\eta) \cos \alpha \quad \psi_{\|} \triangleq \frac{1}{2}(1-\eta) \sin \alpha
$$

and the SRP force components in the $\mathcal{O}^{2}$-frame are

$$
\begin{aligned}
& F_{r}=P A \cos \alpha(1+\eta \cos 2 \alpha) \\
& F_{t}=P A \cos \alpha(\eta \sin 2 \alpha)
\end{aligned}
$$

\section{Sail Performance Parameters}

Within this section, the two most commonly used solar sail performance parameters are introduced. The characteristic acceleration $a_{c}$ is defined as the SRP acceleration acting on a solar sail that is oriented per- 
pendicular to the sun line $\left(\boldsymbol{n} \equiv \boldsymbol{e}_{r}\right)$ at $r_{0}(1 \mathrm{AU})$. For model IR

$$
a_{c}=\frac{2 P_{0} A}{m}
$$

and for model NPR

$$
a_{c}=\frac{2 P_{0} A}{m}\left(a_{1}+a_{2}\right)
$$

The lightness number $\beta$ is defined as the ratio of the SRP acceleration acting on a solar sail that is oriented perpendicular to the sun line $\left(\boldsymbol{n} \equiv \boldsymbol{e}_{r}\right)$, and the gravitational acceleration of the sun, $\mu / r^{2}$ :

$$
\beta=\frac{a_{c}\left(r_{0} / r\right)^{2}}{\mu / r^{2}}=\frac{a_{c}}{\mu / r_{0}^{2}}
$$

with $\mu / r_{0}^{2} \doteq 5.930 \mathrm{~mm} / \mathrm{s}^{2}$ as the sun's gravitational acceleration at Earth distance. Another possible way to define a lightness number is by thinking of it as a dimensionless sail loading, that is, $\beta_{\sigma} \triangleq \sigma^{*} / \sigma$ where $\sigma \triangleq m / A$ is the sail loading and $\sigma^{*} \triangleq 2 P_{0} /\left(\mu / r_{0}^{2}\right)$ is a reference sail loading parameter. As a result, $\beta_{\sigma}$ plays the role of a technological parameter in that it establishes the limits of the current (or future) sail technology. With this definition one gets:

$$
a_{c}=\beta_{\sigma}\left(a_{1}+a_{2}\right) \frac{\mu}{r_{0}^{2}}
$$

or

$$
\beta_{\sigma}=\frac{a_{c}}{\mu / r_{0}^{2}} \frac{1}{a_{1}+a_{2}}=\frac{\beta}{a_{1}+a_{2}}
$$

Equation (24) shows that, in general, $\beta_{\sigma} \neq \beta$. Note, however, that for model IR $a_{1}+a_{2}=1$, and $\beta_{\sigma} \equiv \beta$. To avoid ambiguity, we will use $\beta$ as the lightness number for the remainder of this paper.

\section{The Non-Perfectly Reflecting Solar Sail with Degradation}

\section{A. Data Available From Ground Testing}

Although much ground and space testing has been done to measure the optical degradation of metalized polymer films as second surface mirrors (metalized on the back side), to our knowledge, no systematic testing to measure the optical degradation of candidate solar sail films (metalized on the front side) has been reported so far. Lura et. al. reported in Ref. 11 considerable OSSD after combined irradiation with VUV, electrons, and protons, whereas Edwards et. al. reported in Ref. 12 that a change of the solar absorption and emission coefficients could not be measured after irradiation with electrons alone. Because respective in-space tests have not been made so far, the optical degradation behavior and therefore the degradation behavior of the propulsive capability of solar sails in the real space environment is to a considerable degree indefinite. Nevertheless, solar sail mission designers necessitate an OSSD model to estimate the potential

effects of OSSD on their missions. Therefore, the authors of this paper established in November 2004 a "Solar Sail Degradation Model Working Group" with the aim to elaborate a parametric model for OSSD.

\section{B. Parametric Degradation Model}

For a first OSSD model, the following simplifications may be made:

1. The only source of degradation are the solar photons and particles. This simplification is reasonable at least in the inner solar system and far from the planets, where the cosmic radiation, the radiation from the planetary radiation belts and reactions with particles of the planetary atmospheres can be neglected.

2. The solar photon and particle fluxes do not depend on time (average sun without solar events).

3. The optical coefficients do not depend on the sail temperature.

4. The optical coefficients do not depend on the light incidence angle. 
5. No self-healing effects occur in the sail film.

Let $p$ be an arbitrary optical coefficient from $\mathcal{P}$. With OSSD, $p$ becomes time-dependent, $p(t)$. With the simplifications stated above, $p(t)$ is a function of the solar radiation dose (SRD, dimension $\left[\mathrm{J} / \mathrm{m}^{2}\right]$ ) accepted by the solar sail within the time interval $t-t_{0}$ :

$$
\tilde{\Sigma}(t) \triangleq \int_{t_{0}}^{t} S \cos \alpha d t^{\prime}=S_{0} r_{0}^{2} \int_{t_{0}}^{t} \frac{\cos \alpha}{r^{2}} d t^{\prime}
$$

The symbol $\tilde{\Sigma}$ is used for the SRD, this way preserving $\Sigma$ for the dimensionless SRD. The SRD per year on a surface that is perpendicular to the sun at $1 \mathrm{AU}$ is

$$
\tilde{\Sigma}_{0}=S_{0} \cdot 1 \mathrm{yr}=1368 \mathrm{~W} / \mathrm{m}^{2} \cdot 1 \mathrm{yr}=15.768 \mathrm{TJ} / \mathrm{m}^{2}
$$

Using $\tilde{\Sigma}_{0}$ as a reference value, the SRD can also be defined in a dimensionless form as

$$
\Sigma(t)=\frac{\tilde{\Sigma}(t)}{\tilde{\Sigma}_{0}}=\left(r_{0}^{2} \int_{t_{0}}^{t} \frac{\cos \alpha}{r^{2}} d t^{\prime}\right) / 1 \mathrm{yr}
$$

Because the solar particle flux $S_{\mathrm{pf}}$ also varies with $\cos \alpha / r^{2}$, its (damaging) dose within the time interval $t-t_{0}$ can be written as $\Sigma_{\mathrm{pf}}(t)=\gamma_{\mathrm{pf}} \Sigma(t)$, so that $p(t)=p\left(\Sigma(t)+\Sigma_{\mathrm{pf}}(t)\right)=p\left(\left(1+\gamma_{\mathrm{pf}}\right) \Sigma(t)\right)=p(\Sigma(t))$. Thus, the degradation effects of the solar particles do not have to be considered separately. $\Sigma(t)$ depends on the solar distance history and the attitude history $\boldsymbol{z}[t]=(r, \alpha)[t]$ of the solar sail, $\Sigma(t)=\Sigma(\boldsymbol{z}[t])$. Eq. (27) can also be rearranged in differential form as

$$
\dot{\Sigma}=\frac{r_{0}^{2}}{T} \frac{\cos \alpha}{r^{2}} \quad \text { with } \quad \Sigma\left(t_{0}\right)=0
$$

where $T \triangleq 1$ yr. We assume that $p(t)$ varies exponentially between $p\left(t_{0}\right)=p_{0}$ and $\lim _{t \rightarrow \infty} p(t)=p_{\infty}$ :

$$
p(t)=p_{\infty}+\left(p_{0}-p_{\infty}\right) \cdot \mathrm{e}^{-\lambda \Sigma(t)}
$$

The degradation constant $\lambda$ is related to the "half life solar radiation dose" $\hat{\Sigma}\left(\Sigma=\hat{\Sigma} \Rightarrow p=\frac{p_{0}+p_{\infty}}{2}\right)$ via

$$
\lambda=\frac{\ln 2}{\hat{\Sigma}}
$$

Note that this model has 12 free parameters additional to the $6 p_{0}, 6 p_{\infty}$ and 6 half life SRDs $\hat{\Sigma}_{p}$. Because 12 parameters are too much for a simple parametric OSSD analysis, the number of free parameters should be reduced. This is done here by introducing a degradation factor $d$ and by using a single half life SRD for all $p, \hat{\Sigma}_{p}=\hat{\Sigma} \forall p \in \mathcal{P}$. Because the reflectivity of the sail decreases with time, the sail becomes more matt with time, and the emissivity increases with time, we can use

$$
\begin{aligned}
& \rho_{\infty}=\frac{\rho_{0}}{1+d} \quad s_{\infty}=\frac{s_{0}}{1+d} \quad \varepsilon_{\mathrm{f}_{\infty}}=(1+d) \varepsilon_{\mathrm{f}_{0}} \\
& \varepsilon_{\mathrm{b} \infty}=\varepsilon_{\mathrm{b} 0} \quad B_{\mathrm{f} \infty}=B_{\mathrm{f} 0} \quad B_{\mathrm{b} \infty}=B_{\mathrm{b} 0}
\end{aligned}
$$

The degradation of the optical parameters can again be written in dimensionless form:

$$
\frac{p(t)}{p_{0}}=\left\{\begin{array}{lll}
\left(1+d \mathrm{e}^{-\lambda \Sigma(t)}\right) /(1+d) & \text { for } & p \in\{\rho, s\} \\
1+d\left(1-\mathrm{e}^{-\lambda \Sigma(t)}\right) & \text { for } & p=\varepsilon_{\mathrm{f}} \\
1 & \text { for } & p \in\left\{\varepsilon_{\mathrm{b}}, B_{\mathrm{f}}, B_{\mathrm{b}}\right\}
\end{array}\right.
$$

Fig. 4(a) shows the variation of those coefficients with $\Sigma$ for different degradation factors $d$. Using this model, the $a / \varepsilon_{\mathrm{f}}$-ratio raises from

$$
\frac{a_{0}}{\varepsilon_{\mathrm{f} 0}}=\frac{1-\rho_{0}}{\varepsilon_{\mathrm{f}_{0}}}=2.4
$$




$$
\frac{a_{\infty}}{\varepsilon_{\mathrm{f} \infty}}=\frac{1+d-\rho_{0}}{(1+d)^{2} \varepsilon_{\mathrm{f} 0}}
$$

whose value is 3.1 for a $5 \%$ degradation limit, 3.6 for a $10 \%$ degradation limit, and 4.4 for a $20 \%$ degradation limit (to keep the terminology simple, we will use the term "a 100d\% degradation limit" synonymously for "a degradation factor of $d$ "). Fig. 4(b) shows how the maximum sail temperature $T_{\max }$ varies with $\Sigma$ for different solar distances $0.2 \mathrm{AU} \leq r \leq 1 \mathrm{AU}$. Fig. 5(a) shows the evolution of the $\boldsymbol{F}_{\mathrm{SRP}}$-bubble ${ }^{\mathrm{i}}$ with increasing SRD, and Fig. 5(b) shows the evolution of the difference between the pitch and the cone angle with increasing SRD. Let $F_{t}^{*}=\max _{\alpha} F_{t}$ denote the maximum SRP force component along $\boldsymbol{e}_{t}$ (the direction along which the orbital energy is changed most effectively), $\alpha^{*}$ the pitch angle that yields $F_{t}^{*}$, and the associated cone angle $\theta^{*}$. Fig. 5(c) shows how their values vary with $\Sigma$.
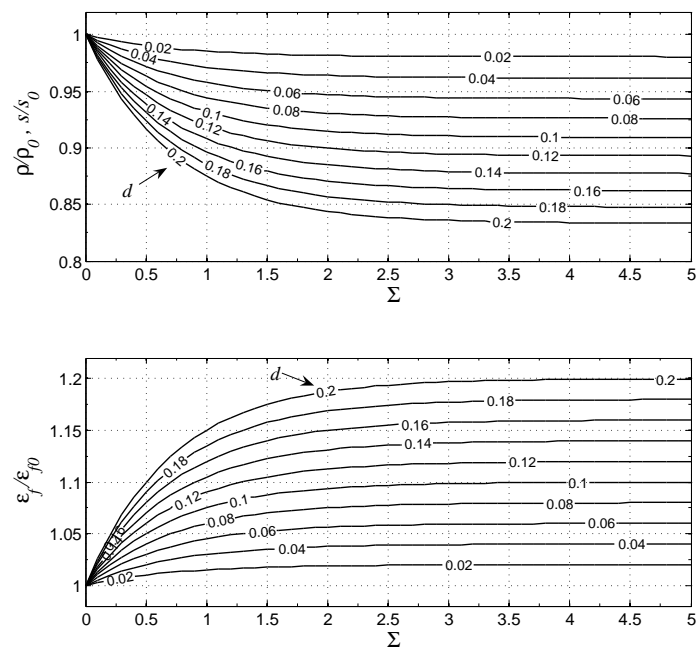

(a) Variation of $\rho / \rho_{0}, s / s_{0}$, and $\varepsilon_{\mathrm{f}} / \varepsilon_{\mathrm{f} 0}$ with $\Sigma$

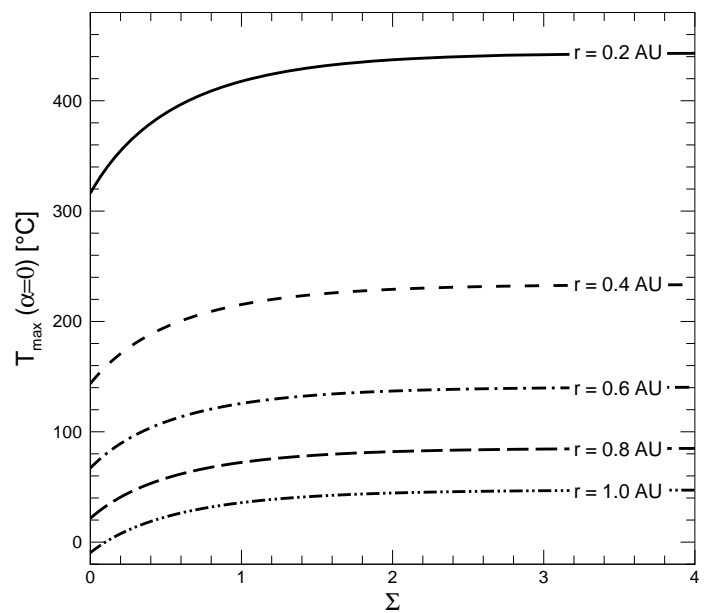

(b) Variation of $T_{\max }$ with $\Sigma$ for different solar distances $(d=0.2)$

Figure 4. OSSD of the optical parameters and its effect on the sail temperature

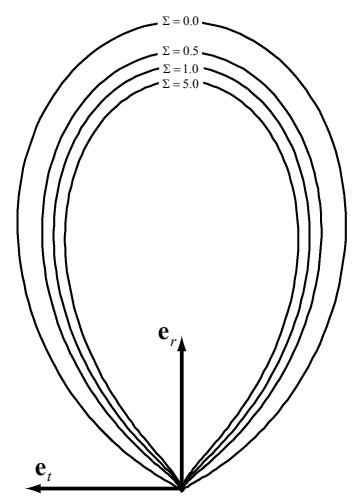

(a) Variation of the $\boldsymbol{F}_{\mathrm{SRP}^{-}}$ bubble with $\Sigma$

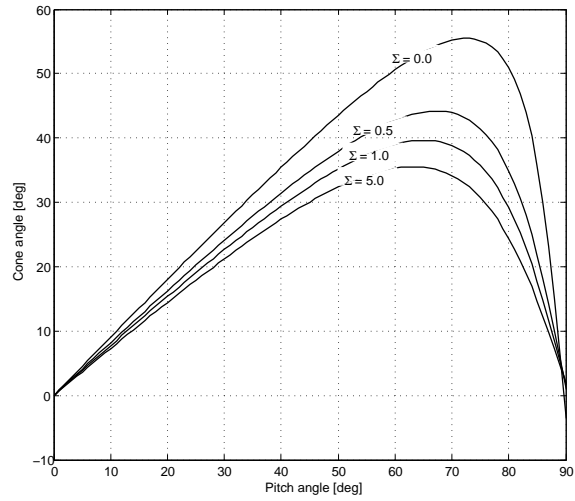

(b) Variation of $\theta$ with $\alpha$ with $\Sigma$
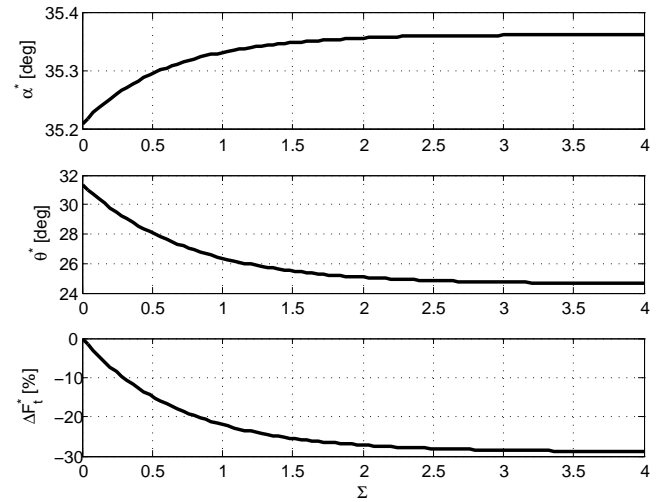

(c) Variation of $\alpha^{*}, \theta^{*}$, and $\Delta F_{t}^{*}$ with $\Sigma$

Figure 5. OSSD effects on attitude control $(d=0.2)$

Note that the OSSD model described here can be directly applied to the generalized sail model (GSM) detailed in Ref. 4. The GSM can model non-flat sails and more complex sail geometries, using the flat plate model given in Eqs. (3)-(5) to define differential sail properties. Because the same optical parameters as given in Eq. (5) are used in the GSM, the effect of optical degradation on the sail can be modeled in the

${ }^{i}$ The bubble on which surface the tip of $\boldsymbol{F}_{\mathrm{SRP}}$ is constrained to lie (tail at origin). 
same way. Thus, it is possible to directly apply this model to the more realistic GSM. For sake of simplicity, such a detailed analysis is not performed in the current paper, but would be of interest for the analysis of a specific sail design.

\section{Potential Degradation Effects on Trajectory and Attitude Control}

\section{A. Equations of Motion and Optimal Control Law}

Besides the gravitational forces of all celestial bodies and the SRP force, many disturbing forces, as caused, e.g., by the solar wind, the finiteness of the solar disk, the reflected light from close celestial bodies, and the aberration of solar radiation (Poynting-Robertson effect), influence the motion of solar sails in space. Furthermore, a real solar sail bends and wrinkles, depending on the actual sailcraft design. Ideally, all these effects have to be considered for high-precision trajectory determination.

For mission feasibility analysis, and to isolate the effects of OSSD from the other effects that influence the motion of real solar sails in space, the following simplifications are made:

1. The solar sail is a flat plate.

2. The solar sail is moving under the sole influence of solar gravitation and radiation.

3. The sun is a point mass and a point light source.

4. The solar sail attitude can be changed instantaneously.

Let the reference frame $\mathcal{I}=\left\{\boldsymbol{e}_{x}, \boldsymbol{e}_{y}, \boldsymbol{e}_{z}\right\}$ be an inertial right-handed cartesian coordinate frame. The equations of motion for a solar sail in the $\mathcal{I}$-frame are:

$$
\begin{aligned}
\dot{\boldsymbol{r}} & =\boldsymbol{v} \\
\dot{\boldsymbol{v}} & =-\frac{\mu}{r^{3}} \boldsymbol{r}+\boldsymbol{a}
\end{aligned}
$$

where $\boldsymbol{r}=\left(r_{x}, r_{y}, r_{z}\right)$ is the sailcraft position and $\boldsymbol{v}=\left(\dot{r}_{x}, \dot{r}_{y}, \dot{r}_{z}\right)=\left(v_{x}, v_{y}, v_{z}\right)$ is the sailcraft velocity, $r=|\boldsymbol{r}|, \mu$ is the sun's gravitational parameter, and $\boldsymbol{a}=\boldsymbol{a}_{\mathrm{SRP}}+\boldsymbol{a}_{d}$ is the acceleration acting on the sailcraft $\left(\boldsymbol{a}_{\mathrm{SRP}}\right.$ being the SRP acceleration and $\boldsymbol{a}_{d}$ being the disturbing acceleration, which is neglected within this paper). Combining Eqs. (13) and (22), the SRP acceleration is given by

$$
\boldsymbol{a}_{\mathrm{SRP}}=\frac{\beta}{b_{1}+b_{2}+b_{3}} \frac{\mu}{r^{2}} \cos \alpha\left[b_{1} \boldsymbol{e}_{r}+\left(b_{2} \cos \alpha+b_{3}\right) \boldsymbol{n}\right]
$$

Although it is very convenient to describe the translational motion of spacecraft in $\mathcal{I}$-frame coordinates, the spacecraft control is better described in the reference frame $\mathcal{O}^{3}=\left\{\boldsymbol{e}_{r}, \boldsymbol{e}_{t}, \boldsymbol{e}_{h}\right\}$. The components of $\boldsymbol{n}$ in the $\mathcal{O}^{3}$-frame are:

$$
\boldsymbol{n}=\cos \alpha \boldsymbol{e}_{r}+\sin \alpha \cos \delta \boldsymbol{e}_{t}+\sin \alpha \sin \delta \boldsymbol{e}_{h}
$$

where $\alpha$ is the sail pitch angle and $\delta$ is the sail clock angle. Clearly, $\alpha$ and $\delta$ comprise the solar sail control $\boldsymbol{u}$. The problem is to find the optimal control law $\boldsymbol{u}(t)$ (where $t \in\left[t_{0}=0, t_{f}\right]$ ), which minimizes the time $t_{f}$ necessary to transfer the spacecraft from an initial state $\boldsymbol{x}_{0}=\left(\boldsymbol{r}_{0}, \boldsymbol{v}_{0}\right)$ to a final state $\boldsymbol{x}_{f}=\left(\boldsymbol{r}_{f}, \boldsymbol{v}_{f}\right)$ by maximizing the performance index $J=-t_{f}$. From Eq. (34), the Hamiltonian of the system is

$$
\mathcal{H}=\boldsymbol{\lambda}_{r} \cdot \boldsymbol{v}-\frac{\mu}{r^{3}} \boldsymbol{\lambda}_{v} \cdot \boldsymbol{r}+\boldsymbol{\lambda}_{v} \cdot \boldsymbol{a}+\lambda_{\Sigma} \frac{r_{0}^{2}}{r^{2} T} \boldsymbol{e}_{r} \cdot \boldsymbol{n}
$$

where $\boldsymbol{\lambda}_{r}$ and $\boldsymbol{\lambda}_{v}$ are the vectors adjoint to the position and the velocity, respectively, and $\lambda_{\Sigma}$ is the costate associated to $\Sigma$. The primer vector ${ }^{13} \boldsymbol{\lambda}_{v}$ can be conveniently expressed in the $\mathcal{O}^{3}$-frame. Paralleling what has been done for $\boldsymbol{n}$, we define the orientation of $\boldsymbol{\lambda}_{v}$ through the angles $\alpha_{\lambda} \in[0, \pi / 2]$ and $\delta_{\lambda} \in[-\pi, \pi]$ to get

$$
\boldsymbol{\lambda}_{v}=\lambda_{v}\left(\cos \alpha_{\lambda} \boldsymbol{e}_{r}+\sin \alpha_{\lambda} \cos \delta_{\lambda} \boldsymbol{e}_{t}+\sin \alpha_{\lambda} \sin \delta_{\lambda} \boldsymbol{e}_{h}\right)
$$

where $\lambda_{v}=\left|\boldsymbol{\lambda}_{v}\right|$. The time derivatives of the adjoint variables are provided by the Euler-Lagrange equations ${ }^{\mathrm{j}}$ :

$$
\dot{\boldsymbol{\lambda}}_{r}=-\frac{\partial \mathcal{H}}{\partial \boldsymbol{r}} \quad \dot{\boldsymbol{\lambda}}_{v}=-\frac{\partial \mathcal{H}}{\partial \boldsymbol{v}} \quad \dot{\lambda}_{\Sigma}=-\frac{\partial \mathcal{H}}{\partial \Sigma}
$$

\footnotetext{
${ }^{\mathrm{j}}$ The explicit form of the partial derivatives is not shown here due to its complexity.
} 
Eq. (39) provides 7 scalar differential equations that must be solved along with the equations of motion, Eqs. (34) and (28). From Pontryagin's maximum principle, the optimal control law $\boldsymbol{u}(t)$, to be selected in the domain of feasible controls $\mathcal{U}$, is such that at any time the Hamiltonian is an absolute maximum. This amounts to maximizing the function $\mathcal{H}^{\prime}$, which coincides with that portion of the Hamiltonian $\mathcal{H}$ that explicitly depends on the control vector, viz.

$$
\boldsymbol{u}=\arg \max _{\boldsymbol{u} \in \mathcal{U}} \mathcal{H} \equiv \arg \max _{\boldsymbol{u} \in \mathcal{U}} \mathcal{H}^{\prime} \quad \text { with } \quad \mathcal{H}^{\prime} \triangleq \boldsymbol{\lambda}_{v} \cdot \boldsymbol{a}+\lambda_{\Sigma} \frac{r_{0}^{2}}{r^{2} T} \boldsymbol{e}_{r} \cdot \boldsymbol{n}
$$

Substituting Eqs. (13), (36), and (38) into Eq. (40) and using the necessary condition $\partial \mathcal{H}^{\prime} / \partial \delta=0$, one has

$$
\tan \delta=\tan \delta_{\lambda}
$$

Eq. (41) states that the unit vectors $\boldsymbol{e}_{r}, \boldsymbol{n}$ and $\boldsymbol{e}_{\boldsymbol{\lambda}_{v}} \triangleq \boldsymbol{\lambda}_{v} / \lambda_{v}$ are coplanar. This generalizes a similar conclusion obtained for model NPR without degradation. ${ }^{10}$ This result is justified by the fact that $\mathcal{H}^{\prime}$ depends on the OSSD model through the term $\lambda_{\Sigma} r_{0}^{2} / r^{2} T \boldsymbol{e}_{r} \cdot \boldsymbol{n}$, which, in turn, is a function of $\alpha$ but not of $\delta$. Concerning the optimal steering law for $\alpha$, an explicit or semi-analytic solution is - in contrast to the ideal and optical force model cases ${ }^{10,14}$ - hardly retrievable. In fact, invoking the necessary condition $\partial \mathcal{H}^{\prime} / \partial \alpha=0$, the following equation is obtained for $\alpha$ :

$$
\begin{aligned}
& b_{1}\left[\sin \left(\alpha+\alpha_{\lambda}\right)+\sin \left(\alpha-\alpha_{\lambda}\right)\right] \\
+ & \frac{b_{2}}{2}\left[2 \sin \left(\alpha-\alpha_{\lambda}\right)+3 \sin \left(3 \alpha-\alpha_{\lambda}\right)+\sin \left(\alpha+\alpha_{\lambda}\right)\right] \\
+ & 2 b_{3} \sin \left(2 \alpha-\alpha_{\lambda}\right)+b_{\Sigma} \sin \alpha=0
\end{aligned}
$$

where

$$
b_{\Sigma} \triangleq \frac{2 r_{0}^{2} \lambda_{\Sigma}}{\beta \mu T \lambda_{v}}\left(b_{1}+b_{2}+b_{3}\right)
$$

The dimensionless variable $b_{\Sigma}$ models the optical degradation of the solar sail. In the ideal case without degradation $\left(\lambda_{\Sigma} \equiv 0 \Rightarrow b_{\Sigma} \equiv 0\right)$, Eq. (42) provides the optimal control law for the optical force model. The corresponding solution is given in Ref. 10. At a generic time $t$, for a given pair $(\beta, d)$, one can calculate the values taken by $b_{\Sigma}, \Sigma, b_{1}, b_{2}$, and $b_{3}$ (which, in turn, depend on $\Sigma, \lambda$, and $d$ ). Let $\mathrm{S}$ be the region of the $\left(\alpha, \alpha_{\lambda}\right)$-plane that satisfies the conditions $\mathcal{H}^{\prime} \geq 0$ and $\partial^{2} \mathcal{H}^{\prime} / \partial \alpha^{2}<0$ (convexity condition). Consider the set of pairs $\left(\widetilde{\alpha}, \widetilde{\alpha_{\lambda}}\right)$ to be solutions of Eq. (42). Note that for a given $\widetilde{\alpha_{\lambda}}$, the corresponding value of $\widetilde{\alpha}$ is obtained through a numerical solution of Eq. (42) (for example using a Newton algorithm). Clearly, $\widetilde{\alpha}$ maximizes $\mathcal{H}^{\prime}$, provided $\left(\widetilde{\alpha}, \widetilde{\alpha_{\lambda}}\right) \in \mathrm{S}$. Otherwise, $\alpha$ should be selected in order to render $\mathcal{H}^{\prime}=0$, that is $\alpha=\pi / 2$. To summarize, the optimal steering law is given by:

$$
\alpha=\left\{\begin{array}{lll}
\widetilde{\alpha} & \text { if } & \mathcal{H}^{\prime}\left(\widetilde{\alpha}, \widetilde{\alpha_{\lambda}}\right) \geq 0 \\
\pi / 2 & \text { if } & \mathcal{H}^{\prime}\left(\widetilde{\alpha}, \widetilde{\alpha_{\lambda}}\right)<0
\end{array}\right.
$$

For a $20 \%$ degradation limit, Fig. 6 shows the optimal steering law for different values of $b_{\Sigma}$ and $\Sigma$ along with the region $\mathbf{S}$ (shaded region). The boundary value problem associated to the variational problem is constituted by the equations of motion, Eqs. (34) and (28), and by the Euler-Lagrange equations, Eq. (39). The boundary conditions are constrained by the planetary ephemerides. They provide 12 scalar conditions connected to the position and velocity of the solar sail at both $t_{0}$ and $t_{f}$. The other boundary condition is given by the initial value of $\Sigma\left(t_{0}\right)=0$. The transversality conditions $\lambda_{\Sigma}\left(t_{f}\right)=0$ and $\mathcal{H}\left(t_{f}\right)=1$ complete the differential problem.

\section{B. Mars Rendezvous}

As the first case to investigate the consequences of OSSD for trajectory and attitude control, the control laws described in section A have been applied to a 2D circular orbit-to-circular orbit Earth-Mars transfer ${ }^{\mathrm{k}}$ using solar sails with various lightness numbers and degradation limits. Like for all trajectories within this paper, direct interplanetary insertion of the solar sail at Earth with zero hyperbolic excess energy $\left(C_{3}=0 \mathrm{~km}^{2} / \mathrm{s}^{2}\right)$

\footnotetext{
${ }^{\mathrm{k}}$ The final position of Mars is left free.
} 

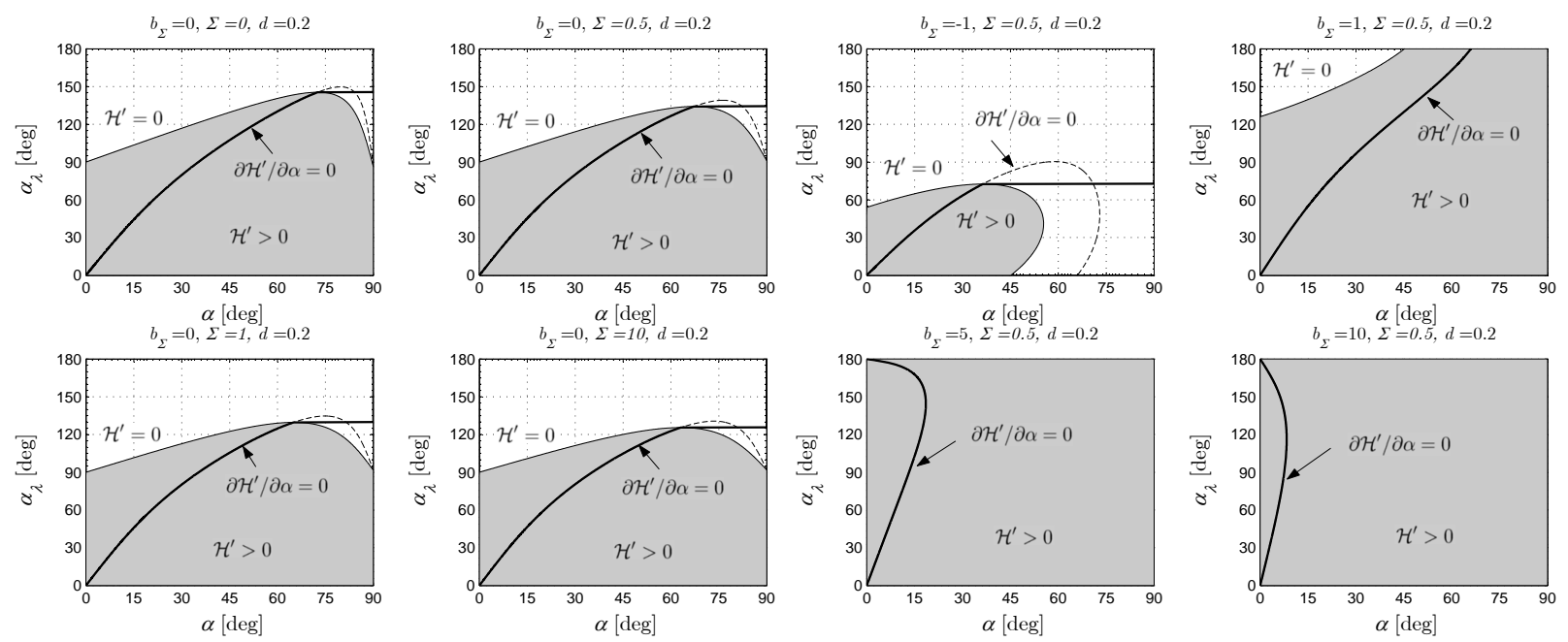

Figure 6. Optimal control law (thick line) for the pitch angle $\alpha$.

is assumed. The trajectories within this section have been calculated using a hybrid numerical technique that combines genetic algorithms to obtain a rough estimate of the adjoint variables with gradient-based and direct methods to refine the solution. ${ }^{10}$ The differential equations have been integrated in double precision using a Runge-Kutta-fifth-order scheme with absolute and relative errors of $10^{-12}$. A set of canonical units ${ }^{15}$ has been used to reduce their numerical sensitivity. The impact of the OSSD model on mission performance has been investigated using three different mathematical models:

Model (a): Within this model, an instantaneous degradation of the optical parameters of the sail is assumed. At $t=t_{0}, \rho, s$, and $\varepsilon_{\mathrm{f}}$ attain immediately their end-of-life values, $\rho_{\infty}, s_{\infty}$, and $\varepsilon_{\mathrm{f} \infty}$. From a mathematical viewpoint, this corresponds to $\hat{\Sigma} \rightarrow 0$ and hence $\lambda \rightarrow \infty$. Accordingly, for a given characteristic acceleration, the obtained performance should be considered as an upper bound for the trip times.

Model (b): Within this model, the degradation of the optical parameters has been taken into account in the numerical integration (that is, in the equations of motion and in the Euler-Lagrange equations), but it has been neglected during the evaluation of the steering law. In other words, the steering law for solar sails without degradation (given in Ref. 10) has also been used for the degrading sails. From a mathematical viewpoint, this corresponds to assuming $b_{\Sigma} \equiv 0$ and $b_{i}(t) \equiv b_{i}\left(t_{0}\right)(i=1,2,3)$ in Eq. (42) only. This model allows to estimate the effect of the OSSD model on the "ideal" control law (that is, on the control law obtained without taking the degradation effect into account). This simplifies the practical implementation of the steering law because the latter is a function of one time-dependent variable only (the primer vector pitch angle $\alpha_{\lambda}$ ).

Model (c): Within this model, the optimal control problem is solved using the OSSD model and the optimal control law described in Eq. (44).

For comparative purposes, also optimal trajectories without degradation have been calculated (these correspond to the model described in Ref. 10). Accordingly, the corresponding results should be considered as a lower bound for the trip times. Fig. 7 summarizes the results obtained for the different models and a degradation limit of $5 \%$ and $20 \%$. The trip times obtained from model (b) are nearly coincident with that from model (c). The main reason is that the values of $b_{\Sigma}$ are small in all simulations (recall that the transversality condition requires $b_{\Sigma}\left(t_{f}\right)=0$ ). As expected, the trip times for model (a) are much greater than that obtained with non-instantaneous degradation. Finally, note that an increase of the degradation factor has remarkable consequences on trip times. This confirms that the degradation of the optical sail characteristics should not be neglected for thorough trajectory calculations and that an ideal model provides excessively optimistic results. 

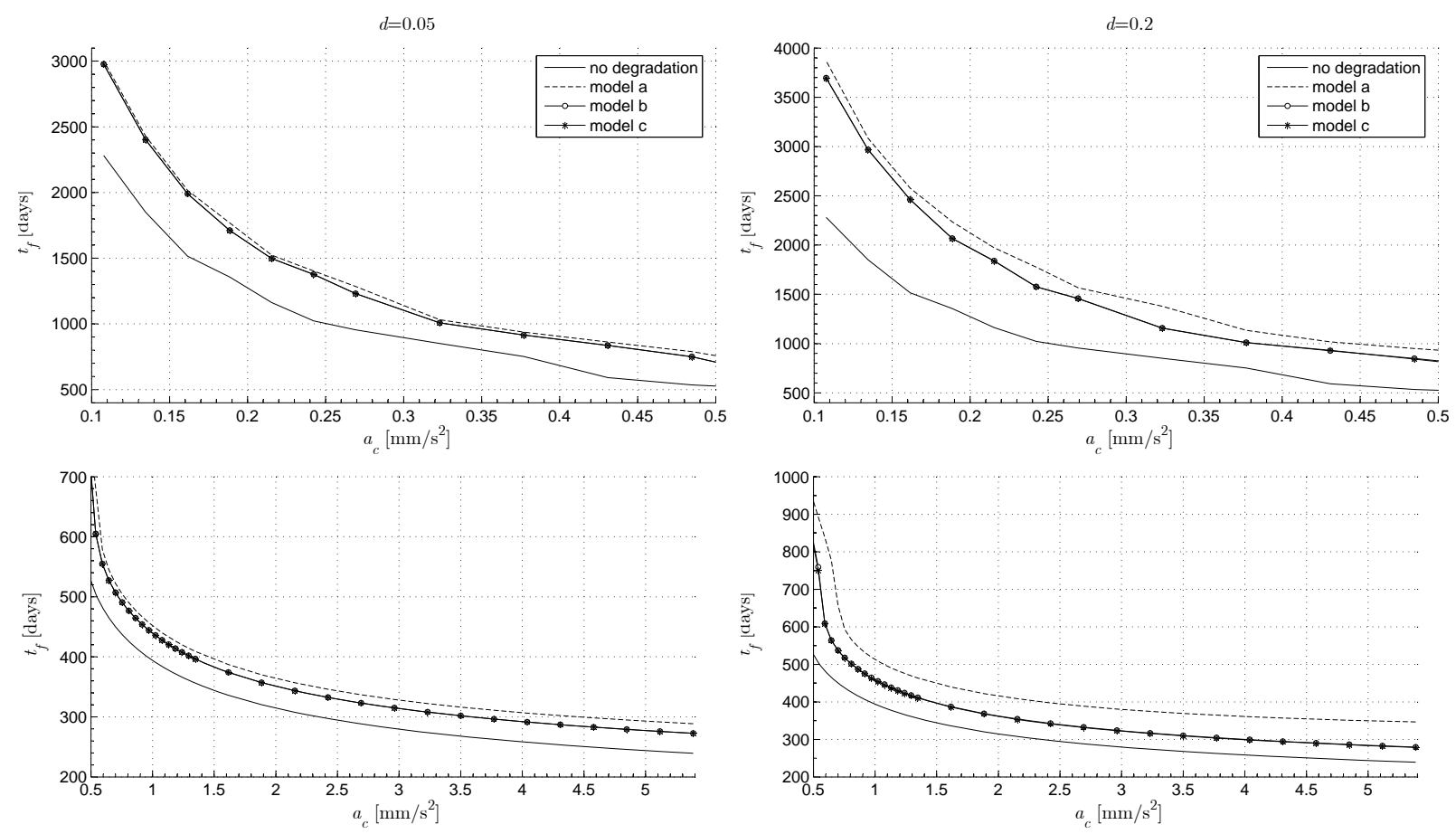

Figure 7. Trip times for an Earth-Mars circular orbit-to-circular orbit transfer for a degradation limit of $5 \%$ and $20 \%(\hat{\Sigma}=0.5)$.

\section{Mercury Rendezvous}

The second case to investigate the consequences of OSSD for trajectory and attitude control is a Mercury rendezvous for a solar sail with a characteristic acceleration of $a_{c}=1.0 \mathrm{~mm} / \mathrm{s}^{2}$. The trajectories within this section have been calculated using the trajectory optimizer GESOP ${ }^{16}$ with SNOPT. ${ }^{17}$ The final accuracy limit for the trajectories was set to $\Delta r_{f, \max }=80000 \mathrm{~km}$ (inside Mercury's sphere of influence at perihelion) and $\Delta v_{f, \max }=50 \mathrm{~m} / \mathrm{s}$. Trajectories have been calculated for degradation limits of $0 \%, 5 \%, 10 \%$, and $20 \%$, with a half life SRD of $\hat{\Sigma}=0.5$. Firstly, the effects of the degradation limit on trip time was investigated for an arbitrarily selected launch window that ranges from MJD 57000 (09 Dec 2014) to MJD 57130 (18 Apr 2015). Fig. 8(a) shows the trip time over the launch date, whereas Fig. 8(b) shows the trip time increase over the launch date. It can be seen from Fig. 8(a) that the sensitivity of the trip time with respect to OSSD depends considerably on the launch date. Some launch dates considered previously as optimal become very unsuitable when OSSD is taken into account because an additional revolution about the sun is required prior to rendezvous. Note that the optimal launch date for zero degradation is the worst launch date for a $20 \%$ degradation limit. For many launch dates, however, OSSD does not seriously affect the mission, as Fig. 8(a) shows. Given an indefinite OSSD behavior at launch, MJD 57000.0 would be a very robust launch date. Fig. 9(a) shows the optimal variation of the pitch angle $\alpha$ along the trajectory for this launch date, Fig. 9(b) for a launch 30 days later. In summary, the results show that OSSD can have remarkable consequences on trip times (Fig. 8) and also on the optimal control angles (Fig. 9). Another important point, which could not be seen from the Mars orbit-to-orbit transfer, is that the actual trip time might be tremendously above the lower bound calculated for orbit-to-orbit transfers, if the launch and the target body are in an unfavorable constellation at launch.

\section{Fast Neptune Flyby}

The third case to investigate the consequences of OSSD for trajectory optimization is a fast Neptune flyby for a solar sail with a characteristic acceleration of $a_{c}=1.0 \mathrm{~mm} / \mathrm{s}^{2}$. The trajectories within this section have been calculated using InTrance, ${ }^{18}$ a method that combines artificial neural networks and evolutionary algorithms to find near-globally optimal steering strategies. To find the absolute trip-time minima, independent of 


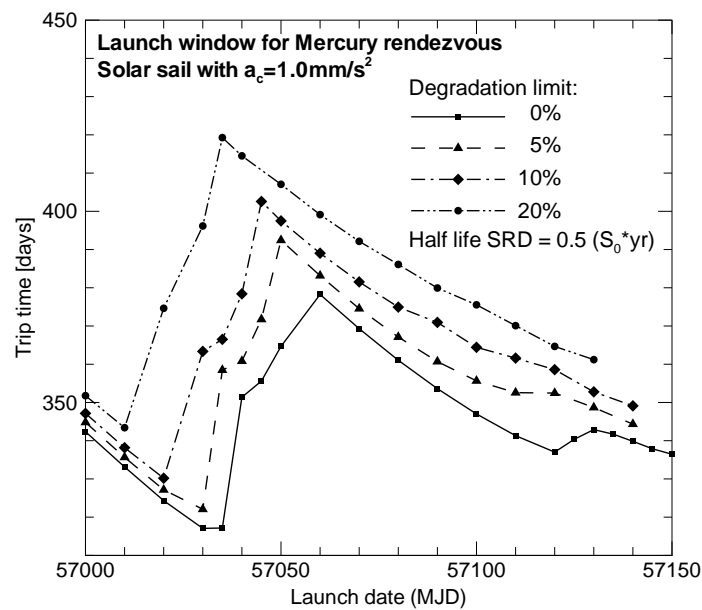

(a) Trip time over launch date

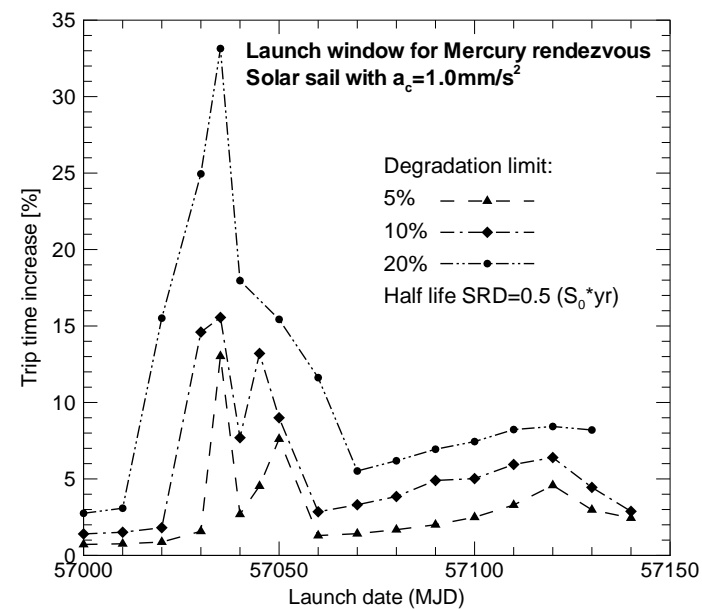

(b) Trip time increase over launch date

Figure 8. Launch window for Mercury rendezvous for different degradation limits

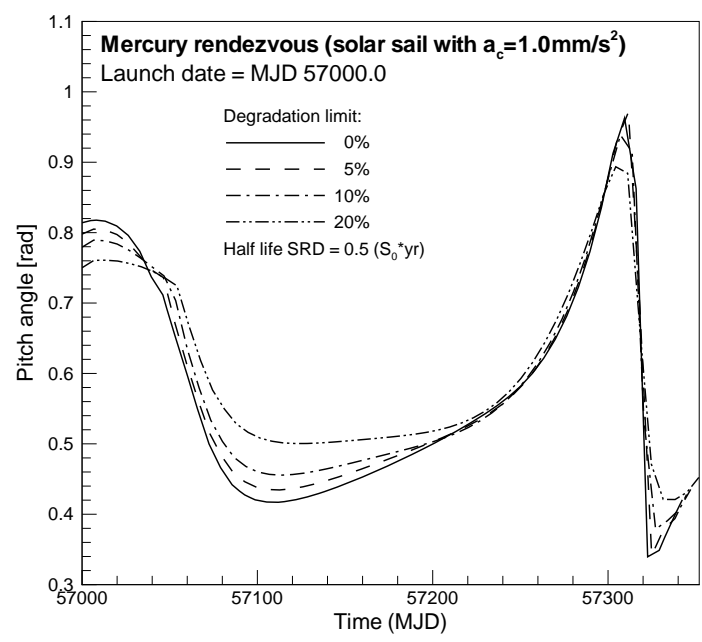

(a) Launch at MJD 57000.0

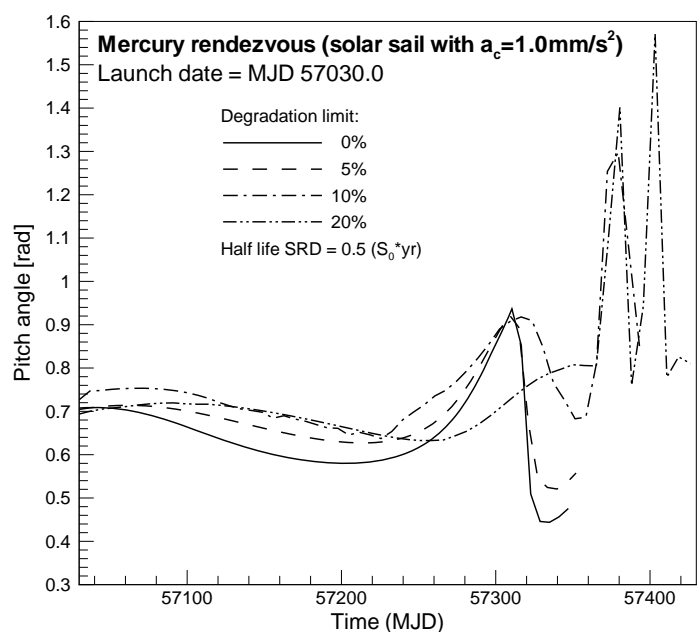

(b) Launch at MJD 57030.0

Figure 9. Optimal sail pitch angle variations for different degradation limits

the actual constellation of Earth and Neptune, no flyby at Neptune itself, but only a crossing of its orbit within a distance of less than $10^{6} \mathrm{~km}$ was required, and InTrance was allowed to vary the launch date within a one year interval. Therefore, the resulting trip times represent lower bounds that are strictly valid only for the optimal constellation of Earth and Neptune. Specific suboptimal launch dates/constellations, e.g., when Neptune is at aphelion, might yield longer trip times. Although a final distance of $10^{6} \mathrm{~km}$ is relatively large for a planetary flyby, the control profile found by InTrance can be used as initial guess for some local trajectory optimization method with a better local convergence behavior. Alternatively, the neurocontroller parameters found by InTrance can be used as initial guess for another run of InTrance (with a more demanding final constraint, e.g. $1000 \mathrm{~km}$ above planetary surface). The sail film temperature was limited to $T_{\text {lim }}=240^{\circ} \mathrm{C}=513.15 \mathrm{~K}$. Fig. 10 shows the trajectories and steering profiles for a $0 \%$ and a $20 \%$ degradation limit. Fig. 11(a) shows the SRD increase and Fig. 11(b) the variation of the solar distance with time. One can see that the aphelion of the last loop becomes larger with an increasing degradation limit and therefore the final solar photonic assist (SPA), i.e., the last close solar approach, occurs later. Fig. 12 shows the trip times for various degradation limits and half life SRDs together with the associated increases. 


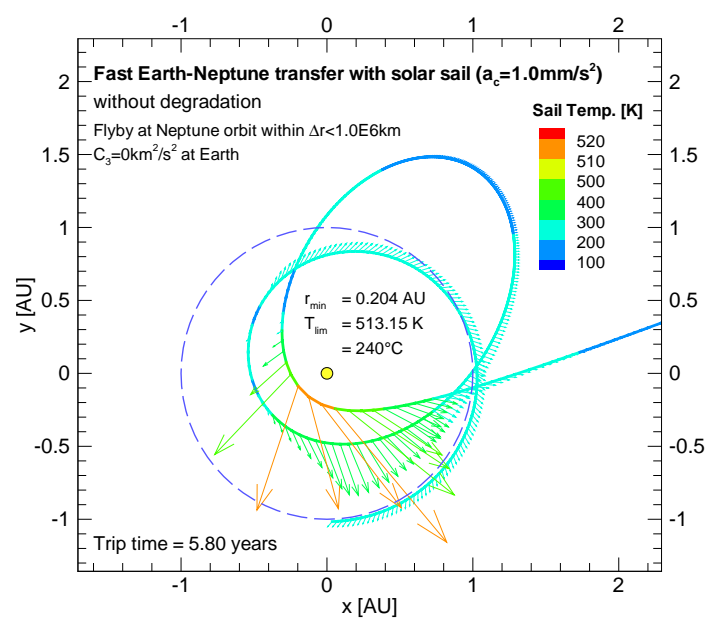

(a)

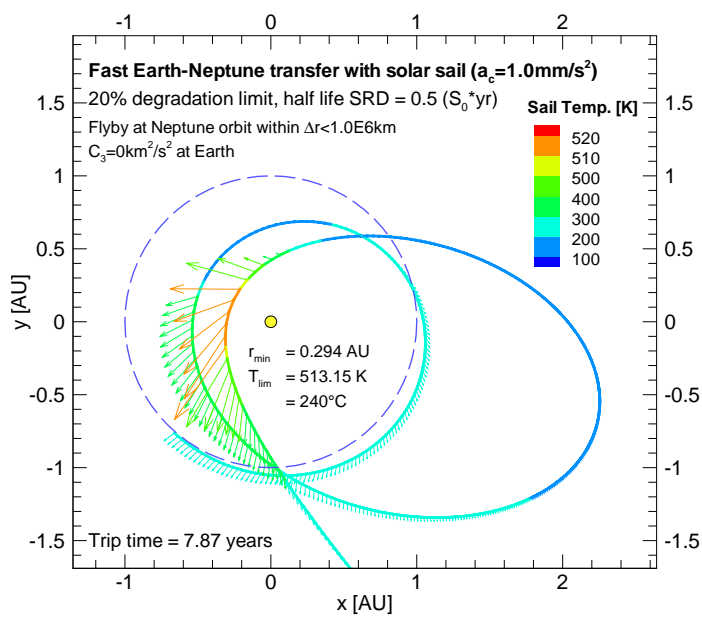

(b)

Figure 10. Topology of optimal Neptune transfer trajectories for different degradation limits

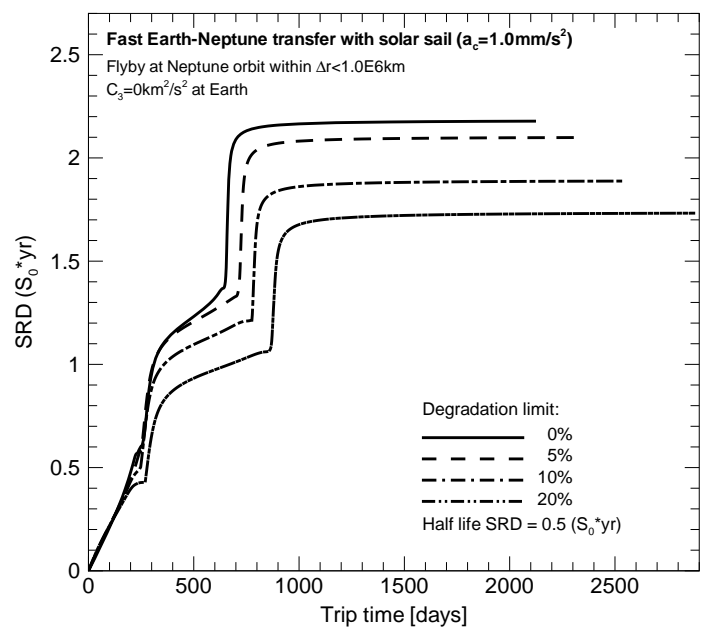

(a) Increase of SRD

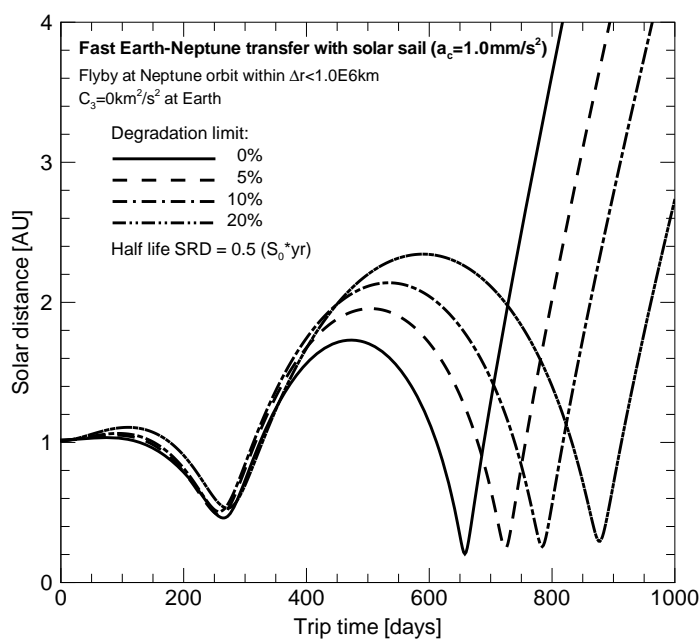

(b) Variation of solar distance

Figure 11. Optimal Neptune transfer for different degradation limits

\section{E. Fast Transfer to the Heliopause}

The fourth case to investigate the consequences of OSSD for trajectory and attitude control is a fast solar sail mission to the heliopause. Following loosely the requirements for ESA's Interstellar Heliopause Probe (IHP) study, ${ }^{19,20}$ the spacecraft should be delivered to the nose of the heliosphere at a latitude of $7.5 \mathrm{deg}$ and a longitude of $254.5 \mathrm{deg}$ (in the ecliptic coordinate frame) at $200 \mathrm{AU}$ from the sun in 25 years. The sail is jettisoned at $5 \mathrm{AU}$ to eliminate any potential interference caused by the solar sail on the local space environment. The trajectories have been calculated using $\mathrm{A}^{\mathrm{n}} \mathrm{D}$ blending, a method that blends locally optimal control laws. ${ }^{21-23}$ Thereby, each control law is prioritized by consideration of how efficiently it will use the SRP and how far each orbital element is from its target value. For non-ideal non-degrading solar sails (model NPR), it has been demonstrated that the trajectories found with $\mathrm{A}^{\mathrm{n}} \mathrm{D}$ blending and InTrance are very similar. ${ }^{21} \mathrm{~A}$ characteristic acceleration of $1.75 \mathrm{~mm} / \mathrm{s}^{2}$ was selected for this analysis, which gives a zero degradation trip time of 21.74 years. For the trajectories within this section a fixed minimum radius of $0.25 \mathrm{AU}$, a half life SRD of $\hat{\Sigma}=0.5\left(S_{0} \cdot \mathrm{yr}\right)$ and a fixed launch date of 03 January 2030 was used. Fig. 13(a) shows the trip time and Fig. 13(b) shows the trip time increase for $0 \leq d \leq 0.3$. For $d \leq 0.2$, the trip time increase is exactly linear. For $d=0.25$ and 0.3 the trip time does not fit the expected linear relationship 


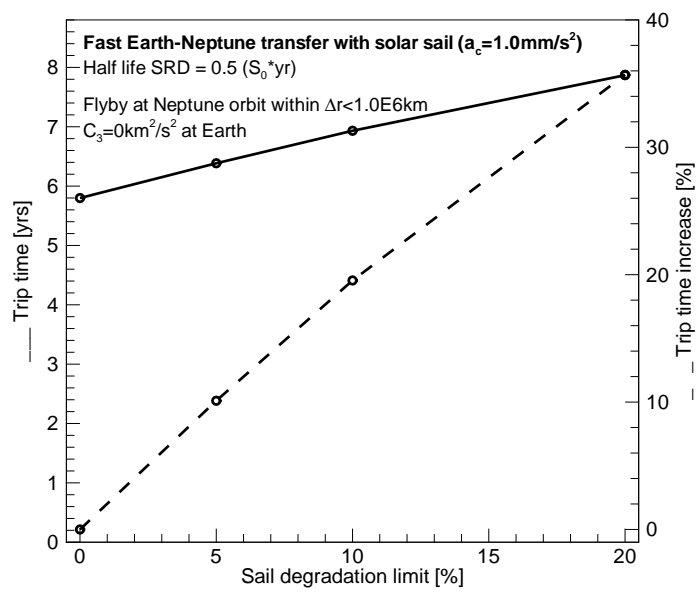

(a) Different degradation limits

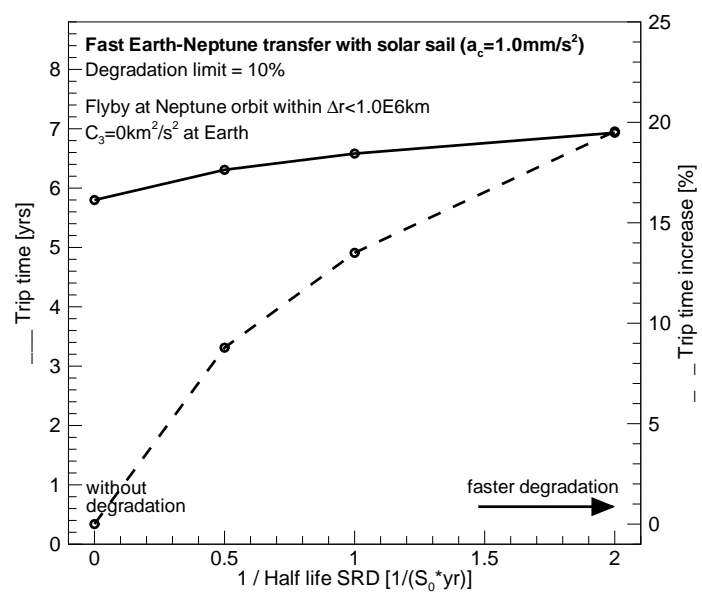

(b) Different half life SRDs

Figure 12. Trip time and trip time increase for optimal Neptune transfer

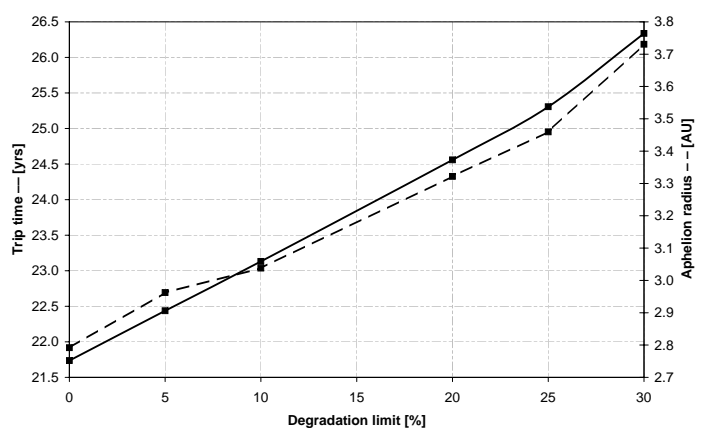

(a) Trip time to $200 \mathrm{AU}$ and radius of aphelion passage

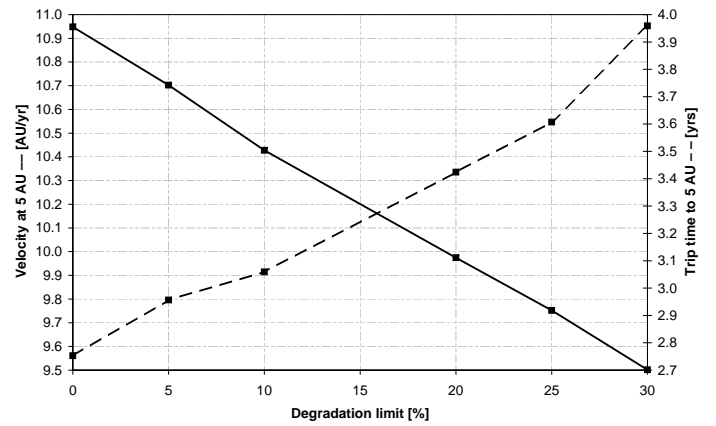

(c) Trip time and velocity at $5 \mathrm{AU}$ (sail jettison point)

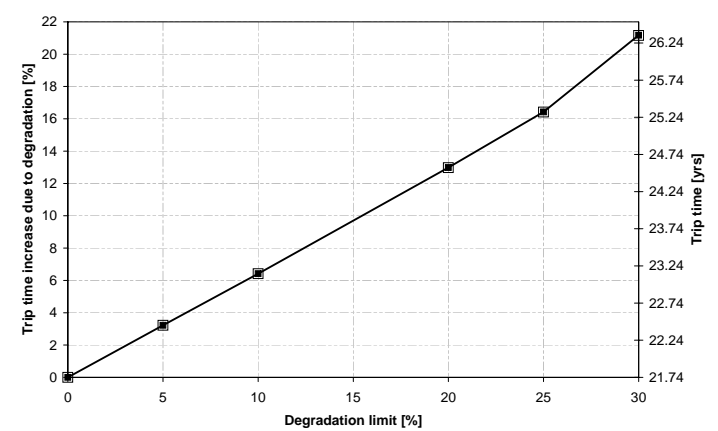

(b) Trip time increase

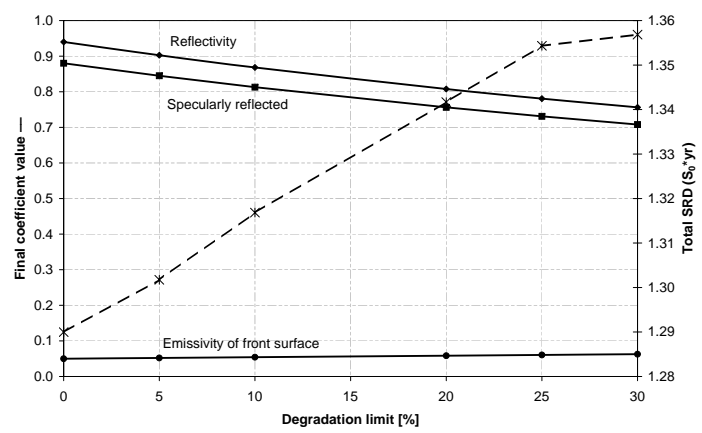

(d) Final optical coefficient values and SRD

Figure 13. Transfer to $200 \mathrm{AU}$ for different degradation limits $(\hat{\Sigma}=0.5)$

as the sail continues to increase the inclination beyond the point where effort would be better spent gaining orbital energy. Fig. 13(a) shows that the radius of aphelion passage increases by over $1 \mathrm{AU}$ as the degradation limit is increased from zero to $30 \%$. Fig. 13(c) shows that the velocity of the spacecraft at sail jettison is decreasing in an approximately linear manner, although the time to sail jettison much more closely matches the shape of the aphelion passage relationship. It is thus notable that given that all these relationships are only approximately linear, the trip time to $200 \mathrm{AU}$ is exactly linear for $0 \leq d \leq 0.2$. Fig. $13(\mathrm{~d})$ shows the value of the optical coefficients at sail jettison and the total SRD increase with degradation limit. Fig. 14(a) shows the most favorable trajectory plot for each degradation limit. Note that a change in degradation limit 


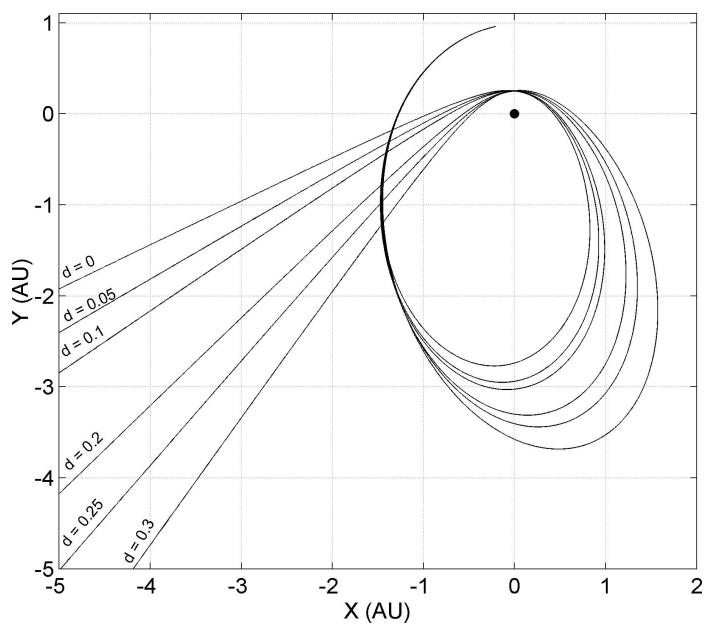

(a) Inner solar system trajectories
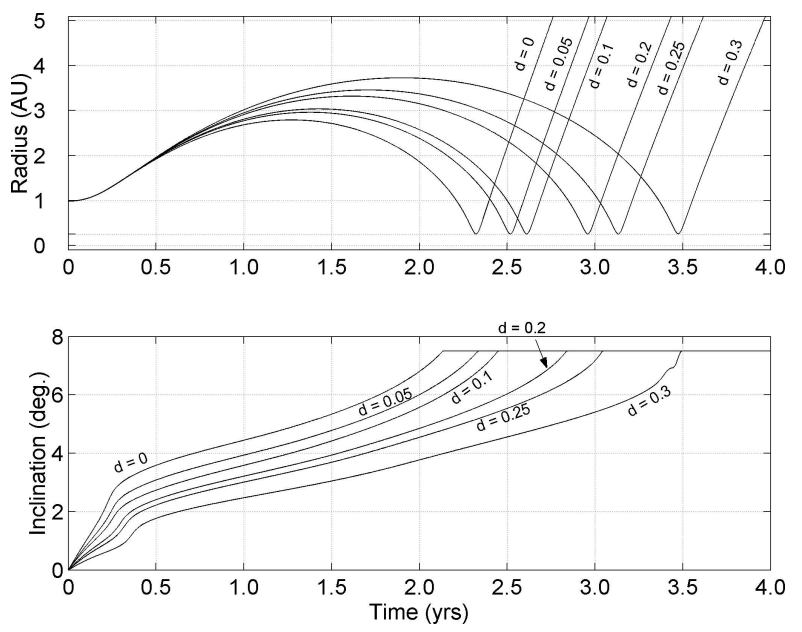

(b) Variation of solar distance and inclination

Figure 14. Transfer to $200 \mathrm{AU}$ for different degradation limits $(\hat{\Sigma}=0.5)$
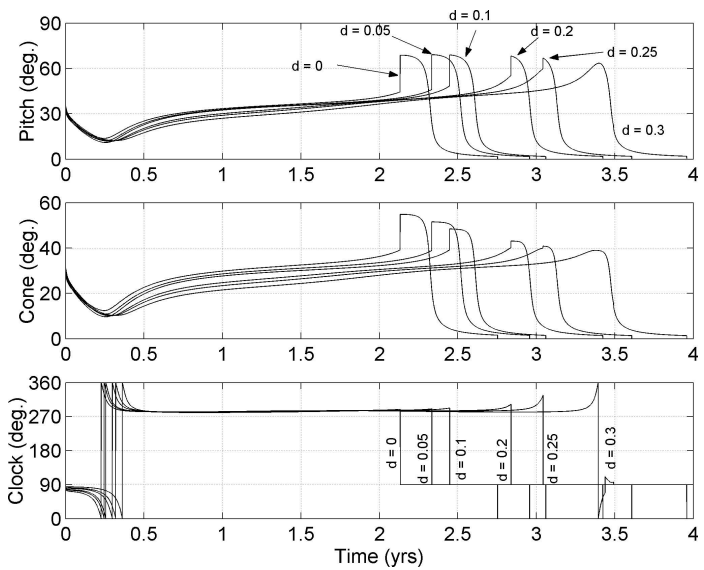

(a) Variation of control angles

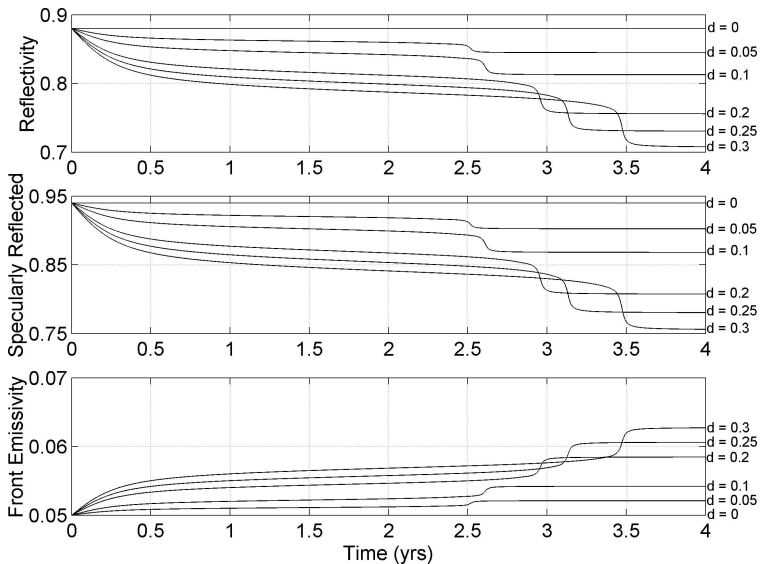

(b) Variation of optical coefficients

Figure 15. Transfer to $200 \mathrm{AU}$ for different degradation limits $(\hat{\Sigma}=0.5)$

for a fixed start epoch significantly changes the final spacecraft azimuth at $200 \mathrm{AU}$, which would significantly impact mission science objectives. In Fig. 14(a) it is seen that the SPA occurs at the same physical location independent of the degradation limit as the argument of pericenter is not varied from trajectory to trajectory, although the time of each SPA is seen to vary by as much as 11/4 years in Fig. 14(b). Fig. 14(b) also shows that for $0 \leq d \leq 0.2$ the inclination has attained its final value significantly before the SPA. At $d=0.25$ the inclination reaches $7.5 \mathrm{deg}$ just prior to the SPA, while at $d=0.3$ this does not occur until after the SPA. The sail control angles used in each best case trajectory are illustrated in Fig. 15(a) where it is noted that the maximum pitch angle of each trajectory is similar, yet the maximum cone angles decreases in-line with the degradation limit increase. Furthermore, the size of the discontinuity within each control angle profile decreases as $d$ increases. Fig. 15(b) shows the variation of the optical coefficients for the trajectories shown in Fig. 14(a), where it is seen that the bulk of the degradation occurs during the close solar pass. It is thus logical to assume that multiple close solar passes would have an adverse effect on the quality of the optical surface and should be avoided when designing such trajectories. 


\section{F. Artificial Lagrange-Point Missions}

This section will examine the artificial Lagrange point problem for solar sails with different reflection coefficients $\eta$ according to the simplified non-perfectly reflecting solar sail model (model SNPR, section II.B). Firstly, equilibrium solutions will be obtained for an ideal solar sail (model IR). Then, the problem will be revisited for solar sails with a reflection coefficient $\eta<1$. Due to the effect that the SRP force vector is no longer directed along $\boldsymbol{n}$, it will be shown that the volume of space available for artificial Lagrange points is extremely sensitive to the reflection coefficient.

\section{Equilibrium Solutions for Model IR}

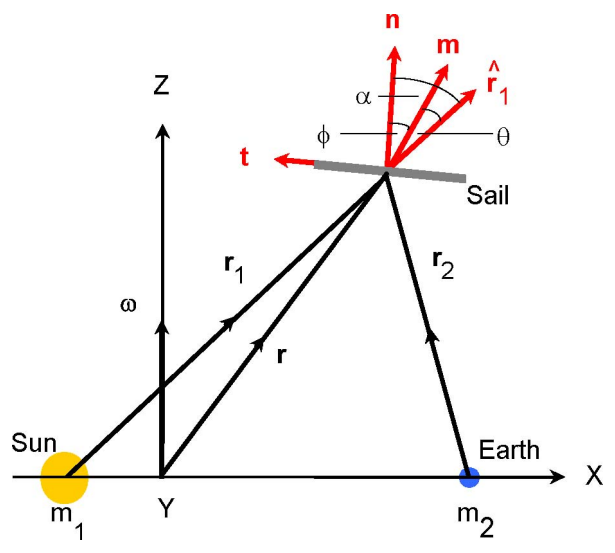

Figure 16. Sun-Earth restricted circular three-body problem with non-perfectly solar sail

Firstly, equilibrium solutions for an ideal sail will be derived. The ideal sail will be considered in a frame of reference co-rotating with the two primary masses $m_{1}$ (Sun) and $m_{2}$ (Earth or other planet) at constant angular velocity $\boldsymbol{\omega}$, as shown in Fig. 16. The sail attitude is defined by the sail normal vector $\boldsymbol{n}$, fixed in the rotating frame of reference. In addition, the ratio of the SRP force to the solar gravitational force exerted on the sail is defined by the sail lightness number $\beta$. Since for $m_{1}>>m_{2}$ both forces have approximately an inverse square variation with solar distance the sail lightness number is nearly a constant ${ }^{1}$. It can be shown that the sail lightness number is related to the total solar sail mass per unit area $\sigma$ by $\sigma=1.53 \mathrm{~g} / \mathrm{m}^{2} / \beta$. Let the unit of mass be chosen such that $\bar{\mu}=G\left(m_{1}+m_{2}\right)=1$. If we now define $\mu=m_{2} /\left(m_{1}+m_{2}\right)$ then in this system the two masses are $\mu_{1}=1-\mu$ and $\mu_{2}=\mu$. The unit of length is chosen such that the constant separation of the two masses is also unity. The vector equation of motion for a solar sail in this rotating frame of reference may be written in standard form as

$$
\ddot{\boldsymbol{r}}+2 \boldsymbol{\omega} \times \dot{\boldsymbol{r}}+\nabla U=\boldsymbol{a}
$$

with the three-body gravitational potential $U$ and the solar radiation pressure acceleration $\boldsymbol{a}$ defined by

$$
\begin{aligned}
U & =-\left[\frac{1}{2}\left(x^{2}+y^{2}\right)+\frac{1-\mu}{r_{1}}+\frac{\mu}{r_{2}}\right] \\
\boldsymbol{a} & =\beta \frac{1-\mu}{r^{2}}\left(\hat{\boldsymbol{r}}_{1} \cdot \boldsymbol{n}\right)^{2} \boldsymbol{n}
\end{aligned}
$$

where the sail position vectors are defined as $\boldsymbol{r}_{1}=(x+\mu, y, z)$ and $\boldsymbol{r}_{2}=(x-(1-\mu), y, z)$. Equilibrium solutions are now required in the rotating frame of reference so that the first two terms of Eq. (45) vanish. The five classical Lagrange points are then obtained as the solutions to $\nabla U=0$ with $\hat{\boldsymbol{r}}_{1} \cdot \boldsymbol{n}=0$ and so $\boldsymbol{a}=0$. However, for $\hat{\boldsymbol{r}}_{1} \cdot \boldsymbol{n}>0$ there is an additional acceleration $\boldsymbol{a}$ which is a function of the lightness number $\beta$ and the attitude $\boldsymbol{n}$ so that new artificial equilibrium solutions may be generated. Since the vector $\boldsymbol{a}$ is oriented in direction $\boldsymbol{n}$, taking the vector product of Eq. (45) with $\boldsymbol{n}$ it follows that

$$
\nabla U \times \boldsymbol{n}=0 \quad \Rightarrow \quad \boldsymbol{n}=\lambda \nabla U
$$

\footnotetext{
${ }^{1}$ Note that the finite size of the solar disc leads to a very small deviation of the SRP from a $1 / r_{1}^{2}$ law.
} 
where $\lambda$ is an arbitrary scalar multiplier. Using $|\boldsymbol{n}|=1, \lambda$ is identified as $1 /|\nabla U|$ so that the required sail attitude is defined by

$$
\boldsymbol{n}=\frac{\nabla U}{|\nabla U|}
$$

which can be used to obtain the pitch angle $\alpha$. The required sail lightness number may also be obtained by taking the scalar product of Eq. (45) with $\boldsymbol{n}$. Again requiring an equilibrium solution it is found that

$$
\beta=\frac{r^{2}}{1-\mu} \frac{\nabla U \cdot \boldsymbol{n}}{\left(\hat{\boldsymbol{r}}_{1} \cdot \boldsymbol{n}\right)^{2}}
$$

Since the sail lightness number and attitude can be selected, the set of five classical Lagrange points will be replaced by an infinite set of artificially generated equilibrium solutions. The regions in which these new solutions may exist are defined by the constraint $\hat{\boldsymbol{r}}_{1} \cdot \nabla U \geq 0$ with a boundary surface defined by an equality. This constraint may be understood physically since the solar radiation pressure acceleration vector $\boldsymbol{a}$, and so the sail normal vector $\boldsymbol{n}$, can never be directed sunward. The boundary surface has two topologically disconnected surfaces $S_{1}$ and $S_{2}$ which define the region of existence of equilibrium solutions near $m_{2}$. The classical equilibrium solutions lie on either $S_{1}$ or $S_{2}$ since they are the solutions to $\nabla U=0$. Surfaces of constant sail lightness number generated from Eq. (49) for the Earth-Sun system are shown in Fig. 17(a). In general, the surfaces of constant sail lightness number approach these boundaries asymptotically with $\beta \rightarrow \infty$ when $\hat{\boldsymbol{r}}_{1} \cdot \boldsymbol{n} \rightarrow 0$ as is clear from Eq. (49). It can be seen that as the sail lightness number increases larger volumes of space are accessible for artificial equilibrium points.

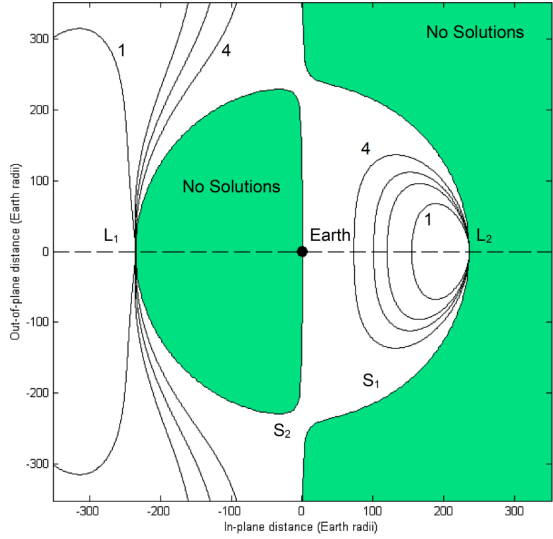

(a) $\eta=1$

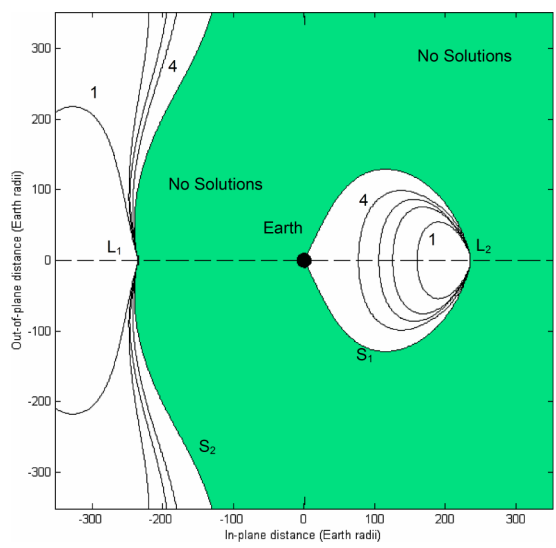

(c) $\eta=0.8$

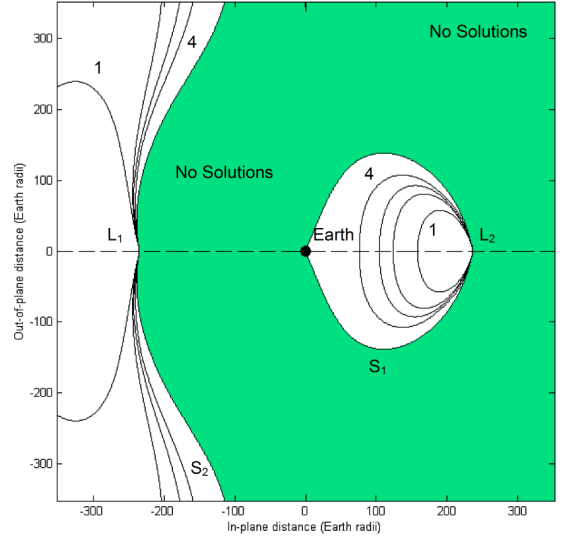

(b) $\eta=0.9$

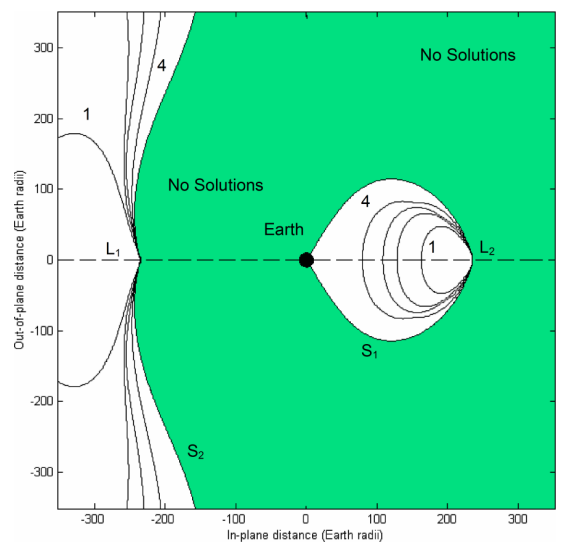

(d) $\eta=0.7$

Figure 17. Contours of sail loading $\sigma$ in the $x$-z-plane: [1] 30 [2] 15 [3] 10 [4] $5\left(\right.$ in $\left.\mathrm{g} / \mathrm{m}^{2}\right)$ 


\section{Equilibrium Solutions for Model SNPR}

Now, model SNPR will be considered, which allows - in contrast to model NPR - a closed-form solution. The SRP acceleration will act now in direction $\boldsymbol{m}$ and may be written as the sum of components normal to the sail surface (along $\boldsymbol{n}$ ) and transverse to the sail surface (along $\boldsymbol{t}$ )

$$
a \boldsymbol{m}=\frac{1}{2} \beta \frac{1-\mu}{r^{2}}(1+\eta)\left(\hat{\boldsymbol{r}}_{1} \cdot \boldsymbol{n}\right)^{2} \boldsymbol{n}+\frac{1}{2} \beta \frac{1-\mu}{r^{2}}(1-\eta)\left(\hat{\boldsymbol{r}}_{1} \cdot \boldsymbol{n}\right)\left(\hat{\boldsymbol{r}}_{1} \cdot \boldsymbol{t}\right) \boldsymbol{t}
$$

where $\left(\hat{\boldsymbol{r}}_{1} \cdot \boldsymbol{n}\right)$ is $\cos \alpha$ and $\left(\hat{\boldsymbol{r}}_{1} \cdot \boldsymbol{t}\right)$ is $\sin \alpha$. The analysis presented in the previous section will now be repeated using the sail force model defined by Eq. (50) so that the equation of motion may now be written as

$$
\ddot{\boldsymbol{r}}+2 \boldsymbol{\omega} \times \dot{\boldsymbol{r}}+\nabla U=a \boldsymbol{m}
$$

For an equilibrium solution the first two terms of Eq. (51) will again vanish so that the sail attitude must be chosen as

$$
\boldsymbol{m}=\frac{\nabla U}{|\nabla U|}
$$

The unit vector $\boldsymbol{m}$ can now be defined by the cone angle $\theta$ between $\hat{\boldsymbol{r}}_{1}$ and $\boldsymbol{m}$ as

$$
\tan \theta=\frac{\left|\hat{\boldsymbol{r}}_{1} \times \nabla U\right|}{\hat{\boldsymbol{r}}_{1} \cdot \nabla U}
$$

In addition, using Eq. (50), the centerline angle can be obtained from the ratio of the transverse and normal accelerations as

$$
\tan \phi=\frac{1-\eta}{1+\eta} \tan \alpha
$$

where the pitch angle $\alpha=\theta+\phi$. Noting that $\boldsymbol{n} \cdot \boldsymbol{t}=0$ and taking a scalar product of Eq. (51) with the unit vector $\boldsymbol{n}$ gives the required sail lightness number as

$$
\beta=\frac{2 r^{2}}{1-\mu} \frac{\nabla U \cdot \boldsymbol{n}}{(1+\eta)\left(\hat{\boldsymbol{r}}_{1} \cdot \boldsymbol{n}\right)^{2}}
$$

The centerline angle may be obtained explicitly by again noting that $\alpha=\theta+\phi$. Then, after some reduction, Eq. (54) yields the centerline angle directly from the cone angle as

$$
\tan \phi=\frac{\eta}{(1+\eta) \tan \theta}\left[1-\sqrt{1-\frac{1-\eta^{2}}{\eta^{2}} \tan ^{2} \theta}\right]
$$

Lastly, using Eq. (55) it is found that the required sail lightness number may be obtained in terms of the lightness number for an ideal solar sail $\tilde{\beta}$ as

$$
\beta=\frac{2}{1+\eta} \frac{\sqrt{1+\tan ^{2} \phi}}{(1-\tan \theta \tan \phi)^{2}} \tilde{\beta}
$$

where $\tilde{\beta}$ is defined by Eq. (49). Therefore, using Eqs. (53), (56) and (57) the sail orientation and sail lightness number required for an artificial equilibrium solution can be obtained. The effect of a non-ideal solar sail and OSSD is shown in Fig. 17 for a $\eta=0.9,0.8$, and 0.7. Firstly, it can be seen that the volume of space available for equilibrium solutions about L2 is significantly reduced. This is due to the centerline angle which limits the direction in which the SRP force vector can be directed. For solutions near L1 the main effect of the non-ideal sail is to displace the equilibrium solutions towards the Earth. This is due to the reduction in the SRP force magnitude rather than the centerline angle. In general, we can state that equilibrium solutions sunward of L1 are not greatly effected by OSSD while equilibrium solutions about L2 are severely restricted.

\section{Summary and Conclusions}

Based on the current standard model for non-perfectly reflecting solar sails, we have developed a parametric model that includes the optical degradation of the sail film due to the erosive effects of the space 
environment. Using this model, we have investigated the effect of different potential degradation behaviors on trajectory and attitude control for various exemplary missions. All our results show that, in general, optical solar sail degradation has a considerable effect on trip times and on the optimal steering profile. For specific launch dates, especially those that are optimal without degradation, this effect can be tremendous. Having demonstrated the potential effects of optical solar sail degradation on future missions, more research on the real degradation behavior has to be done because the degradation behavior of solar sails in the real space environment is to a considerable degree indefinite. To narrow down the ranges of the parameters of our model, further laboratory tests have to be performed. Additionally, before a mission that relies on solar sail propulsion is flown, the candidate solar sail films have to be tested in the relevant space environment. Some near-term missions currently studied in the US and Europe would be an ideal opportunity for testing and refining our degradation model.

\section{References}

${ }^{1}$ Forward, R., "Grey Solar Sails," The Journal of the Astronautical Sciences, Vol. 38, No. 2, 1990, pp. 161-185.

${ }^{2}$ Wright, J., Space Sailing, Gordon and Breach Science Publishers, Philadelphia, 1992.

${ }^{3}$ McInnes, C., Solar Sailing. Technology, Dynamics and Mission Applications, Springer-Praxis Series in Space Science and Technology, Springer-Praxis, Berlin, Heidelberg, New York, Chicester, 1999.

${ }^{4}$ Rios-Reyes, L. and Scheeres, D., "Generalized Model for Solar Sails," Journal of Spacecraft and Rockets, Vol. 42, No. 1, pp. $182-185$.

${ }^{5}$ Sauer, C., "A Comparison of Solar Sail and Ion Drive Trajectories for a Halley's Comet Rendezvous Mission," AAS/AIAA Astrodynamics Conference, Jackson, USA, September 1977, 77-104.

${ }^{6}$ Cichan, T. and Melton, R., "Optimal Trajectories for Non-Ideal Solar Sails," AIAA/AAS Astrodynamics Specialist Conference, Quebec, Canada, August 2001.

${ }^{7}$ Dachwald, B., "Minimum Transfer Times for Nonperfectly Reflecting Solar Sailcraft," Journal of Spacecraft and Rockets, Vol. 41, No. 4, pp. 693-695.

${ }^{8}$ Dachwald, B., "Interplanetary Mission Analysis for Non-Perfectly Reflecting Solar Sailcraft Using Evolutionary Neurocontrol," Astrodynamics 2003, edited by J. de Lafontaine, A. Treder, M. Soyka, and J. Sims, Vol. 116 Part 2 of Advances in the Astronautical Sciences, Univelt, Inc., 2003, pp. 1247-1262.

${ }^{9}$ Dachwald, B., "Optimal Solar Sail Trajectories for Missions to the Outer Solar System," Journal of Guidance, Control, and Dynamics, in press.

${ }^{10}$ Mengali, G. and Quarta, A., "Optimal Three-Dimensional Interplanetary Rendezvous Using Nonideal Solar Sail," Journal of Guidance, Control, and Dynamics, Vol. 28, No. 1, 2005, pp. 173-177.

${ }^{11}$ Lura, F., Hagelschuer, D., Glotov, A., and Tschaly, Y., "Experiments in the Test Facility KOBE for the Investigation of Degradation Effects on Thin Foil Samples for a Solar Sail Mission Concerning the Simultaneous Influence of Space Environment Properties," $22^{\text {nd }}$ Space Simulation Conference, Ellicott City, USA, October 2002.

${ }^{12}$ Edwards, D., Hubbs, W., Stanaland, T., Hollerman, A., and Altstatt, R., "Characterization of Space Environmental Effects on Candidate Solar Sail Material," Photonics for Space Environments VIII, edited by E. Taylor, Vol. 4823, Proceedings of SPIE, 2002, pp. 67-74.

${ }^{13}$ Lawden, D., Optimal Trajectories for Space Navigation, Butterworths, London, 1963.

${ }^{14}$ Sauer, C., "Optimum Solar-Sail Interplanetary Trajectories," AIAA/AAS Astrodynamics Conference, San Diego, USA, August 1976, 76-792.

${ }^{15}$ Seidelmann, P., Explanatory Supplement to the Astronomical Almanac, Dover Books on Mathematics, University Science Books, Sausalito, 1992.

${ }^{16}$ GESOP Website, http://www.gesop.de.

${ }^{17}$ Gill, P., Murray, W., and Saunders, M., "User's Guide for SNOPT 5.3: A FORTRAN Package for Large-Scale Nonlinear Programming," Tech. rep., Stanford University, 1998.

${ }^{18}$ Dachwald, B., Low-Thrust Trajectory Optimization and Interplanetary Mission Analysis Using Evolutionary Neurocontrol, Doctoral thesis, Universität der Bundeswehr München; Fakultät für Luft- und Raumfahrttechnik, 2004.

${ }^{19}$ Lyngvi, A., Falkner, P., Renton, D., v.d.Berg, M., and Peacock, A., "Technology Reference Studies," $55^{\text {th }}$ International Astronautical Congress, Vancouver, Canada, October 2004, IAC-04-U.1.06.

${ }^{20}$ Lyngvi, A., Falkner, P., Kemble, S., Leipold, M., and Peacock, A., "The Interstellar Heliopause Probe," $55^{\text {th }}$ International Astronautical Congress, Vancouver, Canada, October 2004, IAC-04-Q.2.a.06.

${ }^{21}$ Macdonald, M., McInnes, C., and Dachwald, B., "Analytical Control Laws for Heliocentric Solar Sail Orbit Transfers," Journal of Spacecraft and Rockets, submitted.

${ }^{22}$ Macdonald, M. and McInnes, C., "Realistic Earth Escape Strategies for Solar Sailing," Journal of Guidance, Control, and Dynamics, Vol. 28, No. 2, 2005, pp. 315-323.

${ }^{23}$ Macdonald, M. and McInnes, C., "Analytical Control Laws for Planet-Centred Solar Sailing," Journal of Guidance, Control, and Dynamics, in press. 